\title{
Nonabelian Orbifolds and the Boson-Fermion Correspondence
}

\author{
Chongying Dong ${ }^{1}$, Geoffrey Mason ${ }^{2}$ \\ Department of Mathematics, University of California, Santa Cruz, CA 95064, USA
}

Received: 17 June 1993

\begin{abstract}
For a positive integer $l$ divisible by 8 there is a (bosonic) holomorphic vertex operator algebra (VOA) $V_{\Gamma_{1}}$ associated to the spin lattice $\Gamma_{l}$. For a broad class of finite groups $G$ of automorphisms of $V_{\Gamma_{l}}$ we prove the existence and uniqueness of irreducible $g$-twisted $V_{\Gamma_{l}}$-modules and establish the modular-invariance of the partition functions $Z(g, h, \tau)$ for commuting elements in $G$. In particular, for any finite group there are infinitely many holomorphic VOAs admitting $G$ for which these properties hold. The proof is facilitated by a boson-fermion correspondence which gives a VOA isomorphism between $V_{\Gamma_{l}}$ and a certain fermionic construction, and which extends work of Frenkel and others.
\end{abstract}

\section{Introduction}

For the purposes of explaining our results we assume that the reader is familiar with the basic notions of vertex operator algebra (VOA) and twisted modules, as expounded, for example, in [FLM2, FHL, FFR and D2]. More details about these and related ideas are also presented in Sect. 2 below.

In this paper we shall be concerned primarily with two VOAs. The first of these is the VOA $V_{L}$ constructed in the usual way [FLM2] from the root lattice $L$ of type $D_{l}$. If $\mathfrak{g}$ is the simple Lie algebra of type $D_{l}$ and $\hat{\mathfrak{g}}$ the corresponding affine Lie algebra, then $\hat{g}$ has just four level one standard modules, and in [FFR] they are constructed in the fermionic picture. They are denoted $C M^{0}\left(\mathbb{Z}+\frac{1}{2}\right), C M^{1}\left(\mathbb{Z}+\frac{1}{2}\right)$, $C M^{0}(\mathbb{Z}), C M^{1}(\mathbb{Z})$ to indicate that they are constructed as (even or odd) subspaces of certain Clifford modules. The first of these spaces is in fact a VOA isomorphic to $V_{L}$. Here and below we use the notation $C M^{r}(Z), r=0,1, Z=\mathbb{Z}$ or $\mathbb{Z}+\frac{1}{2}$ to denote these modules.

The second VOA of interest to us is that constructed from the spin lattice $\Gamma_{l}$, namely the lattice spanned by the weights of an irreducible spin representation of $g$.

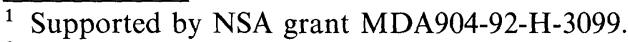

2 Supported by NSF grant DMS-9122030.
} 
If $l$ is divisible by 8 then $\Gamma_{l}$ is an even, self-dual lattice, so that the corresponding VOA $V_{\Gamma_{l}}$ is holomorphic [D1].

We will show that the space $V=C M^{0}\left(\mathbb{Z}+\frac{1}{2}\right) \oplus C M^{0}(\mathbb{Z})$ can be turned into a VOA so that there is an isomorphism of VOAs

$$
\phi: V_{\Gamma_{l}} \rightarrow V .
$$

We call (1.1) the boson-fermion correspondence as it is an extension of the map studied by Frenkel [F] at the level of Kac-Moody algebras.

The major results of the present paper constitute in some sense, an equivariant generalization of (1.1), and are essentially as follows: for a broad class of finite groups $G$ of automorphisms of either $V$ or $V_{L}$ we prove the existence and uniqueness of irreducible $g$-twisted modules $(g \in G)$ and we establish the modular-invariance of the corresponding partition functions. We emphasize that though $G$ is not the most general finite automorphism group, the restrictions placed on $G$ concern its action on $V$ and do not restrict the nature of $G$ itself. Indeed given any finite group $G$, there are infinitely many VOAs $V$ (or $V_{L}$ ) which satisfy our assumptions. So for any finite group $G$ we will have rigorously constructed infinitely many $G$-orbifold theories for which modularinvariance is established.

We should add that although the $g$-twisted modules in question are most easily constructed in the fermionic picture, the vertex operators are best studied in the bosonic picture. Thus the equivariant versions of (1.1), which allow us to pass between the two points-of-view, is a critical component of our method.

For instance, as an example of giving the right answer to the wrong question (!), we construct infinitely many holomorphic VOAs which admit the Monster $M$ as automorphisms, and are such that orbifolding by the Monster yields partition functions which satisfies Norton's axioms [M3, Appendix]. That we cannot solve the problem for the Moonshine module itself is a reflection of the fact that the corresponding twisted sectors are not understood (cf. $[\mathrm{Tu}]$ ).

Let us now give a more precise explanation of our results. Each $C M^{r}(Z)$ is built from an affinization of a space $W \simeq \mathbb{C}^{2 l}$ carrying a non-degenerate symmetric bilinear form $\langle\cdot, \cdot\rangle$. The group $G L(l, \mathbb{C})$ acts on $W$ and preserves a polarization of $W$. Furthermore, $G L(l, \mathbb{C})$ acts on the VOA $C M^{0}\left(\mathbb{Z}+\frac{1}{2}\right)$ as a group of automorphisms. For each $g \in G L(l, \mathbb{C})$ of finite order we show how to construct four $g$-twisted fermionic spaces $C M^{r}(Z, g)$ and we prove that they are, up to isomorphism, the unique irreducible $g$-twisted modules for $V_{L}=C M^{0}\left(\mathbb{Z}+\frac{1}{2}\right)$. Moreover the space $V(g)=C M^{0}\left(\mathbb{Z}+\frac{1}{2}, g\right) \oplus C M^{\varepsilon_{g}}(\mathbb{Z}, g)$ will turn out to be the unique irreducible $g$-twisted $V$-module in case $l$ is divisible by 8 . Here $\varepsilon_{g}=0$ or 1 depends on $g$.

Specializing slightly, if we take $g=S L(l, \mathbb{C})$ then we can take the map (1.1) to be $g$-equivariant. There is a bosonic construction of a $g$-twisted $V_{\Gamma_{l}}$-module which turns out to be an untwisted space $V_{\Gamma_{l, g}+\beta_{g}}$, where $\Gamma_{l, g}$ is a spin lattice associated with $g$ and $\beta \in \mathbb{Q} \otimes \Gamma_{l, g}$. Then (1.1) generalizes to a boson-fermion map

$$
\phi: V_{\Gamma_{l, g}+\beta_{g}} \rightarrow V(g)
$$

which is a $C(g) \cap S L(l, \mathbb{C})$ equivariant identification of the irreducible $g$-twisted $V$-module in the bosonic and fermionic pictures. Furthermore, a second group 
element $h$ induces natural isomorphisms

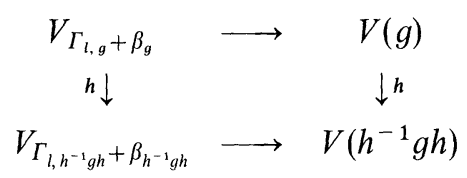

Naturally, there are analogous results for the irreducible $g$-twisted modules of $V_{L}=C M^{0}\left(\mathbb{Z}+\frac{1}{2}\right)$ in the bosonic and fermionic pictures.

We now specialize further, by taking $G<S O(l, \mathbb{R})$ to be a finite group still preserving the polarization of $W$. Under further mild assumptions on the action of $G$ on $W$ we study the partition functions $Z(g, h, \tau)$. Here, $g, h \in G$ are commuting elements and $Z(g, h, \tau)$ is the trace of $h$ on the $g$-twisted $V$-modules $V(g)$ graded by conformal weight with an overall grade-shift determined by the central charge. In case $l$ is divisible by 24 , we show that $Z(g, h, \tau)$ is a modular function, and more precisely satisfies the conditions (which one expects in general)

$$
Z(g, h, \gamma \tau)=\sigma\left(\gamma^{-1}, g, h\right) Z\left(g^{a} h^{c}, g^{b} h^{d}, \tau\right)
$$

for all $\gamma \in\left(\begin{array}{ll}a & b \\ c & d\end{array}\right) \in S L(2, \mathbb{Z})$. Here $\sigma\left(\gamma^{-1}, g, h\right)$ is a certain root of unity and $\tau \in$ $\{z \in \mathbb{C} \mid \operatorname{im} z>0\}$. We emphasize that $G$ can be an arbitrary finite group, so that (1.4) amounts to a proof of modular-invariance for arbitrary finite groups (acting on $W$ in the prescribed manner) of the corresponding fermionic orbifold.

There are corresponding results for the partition functions $Z^{i}(g, h, \tau), 1 \leqq i \leqq 4$ of the four $g$-twisted $C M^{0}\left(\mathbb{Z}+\frac{1}{2}\right)$-modules $C M(Z, g)$. Briefly, if $H$ is the space spanned by the holomorphic partition functions $Z(g, h, \tau)$ above, and if $H_{0}$ is the fourdimensional $S L(2, \mathbb{Z})$-module afforded by the characters of $C M^{r}(Z)[\mathrm{KP}]$ then the space spanned by the $Z^{i}(g, h, \tau)$ turns out to be equivalent, as $S L(2, \mathbb{Z})$-module, to the tensor product $H \otimes H_{0}$.

The proof of modular-invariance is based heavily on the paper [M2]. The spaces constructed there, without the benefit of Lie theory, turns out to be just the spaces $V(g)$ above, and this observation was a strong motivating factor for us. The work of Tsukada [T] was similarly motivated, so that there is a small overlap with his paper. However, he is mainly concerned with superalgebras, as are Feingold et al in Chapter 3 of [FFR]. Namely, $C M^{0}\left(\mathbb{Z}+\frac{1}{2}\right) \oplus C M^{1}\left(\mathbb{Z}+\frac{1}{2}\right)$ is a vertex operator superalgebra, and one can show that $C M^{0}\left(\mathbb{Z}+\frac{1}{2}, g\right) \oplus C M^{1}$ $\left(\mathbb{Z}+\frac{1}{2}, g\right)$ is an irreducible $g$-twisted module and $C M^{0}(\mathbb{Z}, g) \oplus C M^{1}(\mathbb{Z}, g)$ is an irreducible $g z$-twisted module, where $z=\operatorname{diag}(-1, \ldots,-1) \in S L(l, \mathbb{C})$. But the analogue for VOAs seems more difficult, and explains why the maps (1.1), (1.2) are important for us.

The paper is organized as follows: in Sect. 2 we review some definitions, together with a rigorous discussion of projective actions on twisted-modules. In Sect. 3 we construct certain twisted modules in the bosonic picture, whilst in Sect. 4 the analogous construction in the fermionic picture is carried out. Actually, in Sect. 4 we only work at the level of twisted Kac-Moody modules. It is not till Sect. 5 that we are able to establish the full boson-fermion correspondences for the VOA $V_{L}$. Section 6 takes up the same question, but now for the holomorphic VOA $V$; Sect. 7 deals with modular-invariance. 


\section{Vertex Operator Algebras and Modules}

In this section we review the definitions of vertex operator algebras and modules. We define inner automorphisms of a VOA and discuss so-called standard properties of rational VOAs.

A vertex operator algebra (or VOA) is a $\mathbb{Z}$-graded vector space $V=\prod_{n \in \mathbb{Z}} V_{n}$ such that $\operatorname{dim} V_{n}<\infty$ and $V_{n}=0$ if $n$ is sufficiently small, equipped with a linear map

$$
\begin{aligned}
V & \rightarrow(\text { End } V)\left[\left[z, z^{-1}\right]\right], \\
v & \mapsto Y(v, z)=\sum_{n \in \mathbb{Z}} v_{n} z^{-n-1} \quad\left(v_{n} \in \text { End } V\right),
\end{aligned}
$$

and with two distinguished vectors $1 \in V_{0}, \omega \in V_{2}$ satisfying the following conditions for $u, v \in V$ :

$$
\begin{gathered}
u_{n} v=0 \quad \text { for } n \text { sufficiently large; } \\
Y(\mathbf{1}, z)=1 ; \\
Y(v, z) \mathbf{1} \in V[[z]] \text { and } \lim _{z \rightarrow 0} Y(v, z) \mathbf{1}=v ; \\
z_{0}^{-1} \delta\left(\frac{z_{1}-z_{2}}{z_{0}}\right) Y\left(u, z_{1}\right) Y\left(v, z_{2}\right)-z_{0}^{-1} \delta\left(\frac{z_{2}-z_{1}}{-z_{0}}\right) Y\left(v, z_{2}\right) Y\left(u, z_{1}\right) \\
=z_{2}^{-1} \delta\left(\frac{z_{1}-z_{0}}{z_{2}}\right) Y\left(Y\left(u, z_{0}\right) v, z_{2}\right)
\end{gathered}
$$

(Jacobi identity) where $\delta(z)=\sum_{n \in \mathbb{Z}} z^{n}$ is the algebraic formulation of the $\delta$-function at 1 , and all binomial expressions are to be expanded in nonnegative integral powers of the second variable;

$$
[L(m), L(n)]=(m-n) L(m+n)+\frac{1}{12}\left(m^{3}-m\right) \delta_{m+n, 0}(\operatorname{rank} V)
$$

for $m, n \in \mathbb{Z}$, where

$$
L(n)=\omega_{n+1} \quad \text { for } n \in \mathbb{Z} \text {, i.e., } Y(\omega, z)=\sum_{n \in \mathbb{Z}} L(n) z^{-n-2}
$$

and

$$
\begin{gathered}
\operatorname{rank} V \in \mathbb{Q} \\
L(0) v=n v=(\text { wt } v) v \quad \text { for } v \in V_{n}(n \in \mathbb{Z}) \\
\frac{d}{d z} Y(v, z)=Y(L(-1) v, z)
\end{gathered}
$$

This completes the definition. Note that (2.6) says that the operators $L(n)$ generate a copy of the Virasoro algebra, represented on $V$ with central charge rank $V$. We denote the vertex operator algebra just defined by $(V, Y, \mathbf{1}, \omega)$ (or briefly, by $V$ ). The series $Y(v, z)$ are called vertex operators.

Now suppose that $\left(V_{i}, Y_{i}, \mathbf{1}_{i}, \omega_{i}\right)$ are vertex operator algebras, $i=1,2$. A homomorphism from $V_{1}$ to $V_{2}$ is a linear map $f: V_{1} \rightarrow V_{2}$ such that $f\left(\mathbf{1}_{1}\right)=\mathbf{1}_{2}$, $f\left(\omega_{1}\right)=\omega_{2}$ and

$$
f Y_{1}(v, z)=Y_{2}(f(v), z) f
$$


for all $v \in V_{1}$. If $f$ is invertible, or if in addition $\left(V_{1}, Y_{1}, \mathbf{1}_{1}, \omega_{1}\right)=\left(V_{2}, Y_{2}, \mathbf{1}_{2}, \omega_{2}\right)$, we call $f$ an isomorphism or automorphism respectively.

Let $g$ be an automorphism of the VOA $V$ of order $N$. Following [FFR] and [D2], a $g$-twisted module $M$ for $V$ is a $\mathbb{Q}$-graded vector space $M=\prod_{n \in \mathbb{Q}} M_{n}$ such that $\operatorname{dim} M_{n}<\infty$ and $M_{n}=0$ for $n$ is sufficiently small. Moreover there is a linear map

$$
\begin{aligned}
& V \rightarrow(\text { End } M)\left[\left[z^{1 / N}, z^{-1 / N}\right]\right], \\
& v \mapsto Y_{g}(v, z)=\sum_{n \in \frac{1}{N} \mathbb{Z}} v_{n} z^{-n-1} \quad\left(v_{n} \in \text { End } M\right)
\end{aligned}
$$

satisfying axioms analogous to (2.2)-(2.3) and (2.5)-(2.10). To describe these, let $\eta=e^{2 \pi i / N}$ and set $V^{j}=\left\{v \in V \mid g v=\eta^{j} v\right\}, 0 \leqq j \leqq N-1$. Thus we have a direct sum decomposition

$$
V=\prod_{j \in \mathbb{Z} / N \mathbb{Z}} V^{j}
$$

Then we require that for $u, v \in V, w \in M$,

$$
\begin{gathered}
Y_{g}(v, z)=\sum_{n \in j / N+\mathbb{Z}} v_{n} z^{-n-1} \quad \text { for } v \in V^{j} \\
u_{n} w=0 \quad \text { for } n \text { sufficiently large; } \\
\qquad Y_{g}(\mathbf{1}, z)=1 ; \\
z_{0}^{-1} \delta\left(\frac{z_{1}-z_{2}}{z_{0}}\right) Y_{g}\left(u, z_{1}\right) Y_{g}\left(v, z_{2}\right)-z_{0}^{-1} \delta\left(\frac{z_{2}-z_{1}}{-z_{0}}\right) Y_{g}\left(v, z_{2}\right) Y_{g}\left(u, z_{1}\right) \\
=z_{2}^{-1} \frac{1}{N} \sum_{j \in \mathbb{Z} / N \mathbb{Z}} \delta\left(\eta^{j} \frac{\left(z_{1}-z_{0}\right)^{1 / N}}{z_{2}^{1 / N}}\right) Y_{g}\left(Y\left(g^{j} u, z_{0}\right) v, z_{2}\right) .
\end{gathered}
$$

Finally, (2.6)-(2.10) go over unchanged except that in (2.9) we replace $v$ by $w \in M$. This completes the definition. We denote this module by $\left(M, Y_{g}\right)$, or briefly by $M$.

Remark 2.1. In view of (2.13) and the linearity of $Y_{g}$, we may replace (2.17) by the version in which $u$ lies in an eigenspace $V^{k}$. Then (2.17) takes a simpler form

$$
\begin{gathered}
z_{0}^{-1} \delta\left(\frac{z_{1}-z_{2}}{z_{0}}\right) Y_{g}\left(u, z_{1}\right) Y_{g}\left(v, z_{2}\right)-z_{0}^{-1} \delta\left(\frac{z_{2}-z_{1}}{-z_{0}}\right) Y_{g}\left(v, z_{2}\right) Y_{g}\left(u, z_{1}\right) \\
=z_{2}^{-1}\left(\frac{z_{1}-z_{0}}{\mathrm{z}_{2}}\right)^{-K / N} \delta\left(\frac{z_{1}-z_{0}}{z_{2}}\right) Y_{g}\left(Y\left(u, z_{0}\right) v, z_{2}\right) .
\end{gathered}
$$

Remark 2.2. Taking $g=1$ in the foregoing, we obtain the definition of a $V$-module in the precise sense of [FLM2].

Suppose that $\left(M_{i}, Y_{i}\right)$ are two $g$-twisted modules for the VOA $V, i=1,2$. A homomorphism from $M_{1}$ to $M_{2}$ is a linear map $f: M_{1} \rightarrow M_{2}$ which satisfies

$$
f Y_{1}(v, z)=Y_{2}(v, z) f
$$

for all $v \in V$. One can define the notions of $g$-twisted submodules, irreducible $g$-twisted module, isomorphism of $g$-twisted modules etc., in the obvious way. One writes $f:\left(M_{1}, Y_{1}\right) \rightarrow\left(M_{2}, Y_{2}\right),\left(M_{1}, Y_{1}\right) \simeq\left(M_{2}, Y_{2}\right)$. 
Let $V$ be a VOA with automorphisms $g, h$ and with $g$ of finite order, and let $\left(M, Y_{g}\right)$ be a $g$-twisted module for $V$. We can $t$ wist $M$ by $h$ in the usual way to obtain an $h^{-1} g h$-twisted module for $V$; the resulting module is denoted by $\left(h \circ M,{ }_{h} Y_{g}\right)$. By definition, the underlying space $h \circ M$ is the space $M$ itself and if $v \in V$ we define

$$
{ }_{h} Y_{g}(v, z)=Y_{g}(h v, z) .
$$

One readily calculates that this indeed turns $h \circ M$ into an $h^{-1} g h$-module for $V$.

We define an automorphism $h$ of $V$ to be an inner automorphism in case the following condition holds: for each irreducible $V$-module $\left(M, Y_{1}\right)$ we have $\left(M, Y_{1}\right) \simeq\left(h \circ M, Y_{h}\right)$. Obviously, the set of all inner automorphisms of $V$ is a subgroup of the full group of automorphisms of $V$, which we denote by $\operatorname{Inn}(V)$.

It is an important, though standard, consequence that there is a projective representation of $\operatorname{Inn}(V)$ induced on each irreducible $V$-module, and it will be worthwhile to recall the construction here. Let $h \in \operatorname{Inn}(V)$ with $\left(M, Y_{1}\right)$ an irreducible $V$-module. By definition there is an invertible linear map $\phi(h): M \rightarrow M$ satisfying

$$
\phi(h) Y_{1}(v, z) \phi(h)^{-1}=Y_{1}(h v, z)
$$

for all $v \in V$. Then $\phi(h)^{-1} \phi(g)^{-1} \phi(g h)$ commutes with all $Y_{1}(v, z)$ and hence is a scalar $c(g, h)$ since $\left(M, Y_{1}\right)$ is irreducible. So $\phi(g h)=c(g, h) \phi(g) \phi(h)$ for all $g, h \in \operatorname{Inn}(V)$, which is precisely the condition for the map $g \mapsto \phi(g)$ to be a projective representation of $\operatorname{Inn}(V)$ corresponding the class of the 2-cocycle $c(g, h)$ in $H^{2}\left(\operatorname{Inn}(V), \mathbb{C}^{*}\right)$.

The notion of complete-reducibility of $g$-twisted modules being self-evident, we say that a VOA $V$ is rational in case it has only a finite number of (isomorphism classes of) irreducible modules and if every $V$-module is completely reducible; and $V$ is called simple if $V$ is itself an irreducible $V$-module. We call $V$ holomorphic if $V$ is simple, rational and if $V$ is the unique irreducible $V$-module. Clearly, any automorphism of a holomorphic VOA is inner.

We list next so-called "standard" properties of rational VOAs and their twisted modules. Some of these are explicitly or implicitly in the literature and we are confident that they should hold in general; others are more speculative. In any case, we will show that the VOAs which we study in this paper do indeed enjoy these properties.

For the property $\mathrm{SH} 1-\mathrm{SH} 3$ we assume that $V$ is a holomorphic VOA and $G$ is a finite group of automorphisms of $V$ (necessarily inner).

SH1: For each $g \in G$ there is a unique irreducible $g$-twisted module for $V$, call it $M(g)$.

This being the case, the argument following (2.21) applies to the centralizer of $g$, $C_{G}(g)$, and shows that it has a projective representation on $M(g)$, which we denote by $h \mapsto \phi(h)$ as before for $h \in C_{G}(g)$.

SH2: For $h \in C_{G}(g)$ define

$$
Z(g, h, \tau)=\left.\operatorname{tr}\left(\phi(h) q^{L(0)-c / 24}\right)\right|_{M(g)}=q^{-c / 24} \sum_{n \in \mathbb{Q}} \operatorname{tr}\left(\phi(h), M(g)_{n}\right) q^{n},
$$

where $\tau$ is in the complex upper-half plane, $q=e^{2 \pi i \tau}$ and $c=\operatorname{rank} V$. Then $Z(g, h, \tau)$ is a modular function on some congruence subgroup of the modular group.

To clarify what (2.22) means, note that $C_{G}(h)$ acts projectively on each $M(g)_{n}$ (via the same 2-cocycle). Thus really $Z(g, h, \tau)$ is defined only up to a choice of some root of unity. 

SH3: If $g, h \in G$ commute then for any $\gamma=\left(\begin{array}{ll}a & b \\ c & d\end{array}\right)$ in the modular group $S L(2, \mathbb{Z})$
we have

$$
Z(g, h, \gamma \tau)=\sigma\left(\gamma^{-1}, g, h\right) Z\left(g^{a} h^{c}, g^{b} h^{d}, \tau\right)
$$

for some root of unity $\sigma\left(\gamma^{-1}, g, h\right)$.

This is the axiom of Norton (cf. [M3, DGH]).

Now take $V$ to be a rational VOA with $G$ a finite group of inner automorphisms of $V$. The first two properties generalize $\mathrm{SH} 1$ :

SR1: Suppose that $V$ has exactly $N$ (isomorphism classes of) irreducible modules. Then for $g \in G, V$ has exactly $N$ irreducible $g$-twisted modules.

SR2: The irreducible $g$-twisted modules can be indexed $\left(M^{1}(g), Y_{g}^{1}\right), \ldots$, $\left(M^{N}(g), Y_{g}^{N}\right)$ in such a way that for all $h \in G$ we have

$$
\left(h \circ M^{i}(g),{ }_{h} Y_{g}^{i}\right) \simeq\left(M^{i}\left(h^{-1} g h\right), Y_{h^{-1} g h}^{i}\right) .
$$

Once again this would yield a projective representation of $C_{G}(g)$ denoted by $h \mapsto \phi(h)$.

SR3: Let $Z^{i}(g, h, \tau)=\left.\operatorname{tr}\left(\phi(h) q^{L(0)-c / 24}\right)\right|_{M^{\prime}(g)}$ as in (2.22). Then $Z^{i}(g, h, \tau)$ is a modular function.

SR4: There is an integer $l$ such that whenever $g, h \in G$ commute and $\gamma=\left(\begin{array}{ll}a & b \\ c & d\end{array}\right)$ lies in the principal congruence subgroup $\Gamma(l)$ of $S L(2, \mathbb{Z})$, then $(2.23)$ holds for $Z^{i}(g, h, \gamma \tau)$. Moreover for general $\gamma \in S L(2, \mathbb{Z})$ and each $i$ there are coefficients $a_{i j}$ depending on $\gamma, g$ and $h$ such that

$$
Z^{i}(g, h, \gamma \tau)=\sum_{j=1}^{N} a_{i j} Z^{j}\left(g^{a} h^{c}, g^{b} h^{d}, \tau\right)
$$

These are the main properties we shall consider in this paper, but there are a number of other closely related to these: for example the relationship between the fixed-point VOA $V^{G}$ and the $g$-twisted $V$-modules, the relationship with the quantum double $D G$, and the precise nature of the representation of $\Gamma$ on the space of functions $Z^{i}(g, h, \tau)$. For these and other aspects of the theory see, for example, [DVVV, Ba1-Ba2].

It is useful to formulate certain properties of twisted modules in terms of operator-valued rational functions. Let $S$ denote the set of nonzero linear polynomials in variables $z_{1}$ and $z_{2}$ :

$$
S=\left\{c z_{1}+d z_{2}|c, d \in \mathbb{C},| c|+| d \mid \neq 0\right\} \subset \mathbb{C}\left[z_{1}, z_{2}\right]
$$

and consider the subring $\mathbb{C}\left[z_{1}, z_{2}\right]_{s}$ of the field of rational functions $\mathbb{C}\left(z_{1}, z_{2}\right)$ obtained by localizing at $S$. Let $\left(i_{1} i_{2}\right)$ be a permutation of $\{1,2\}$. We define a map

$$
l_{i_{1} i_{2}}: \mathbb{C}\left[z_{1}, z_{2}\right]_{s} \rightarrow \mathbb{C}\left[\left[z_{1}, z_{1}^{-1}, z_{2}, z_{2}^{-1}\right]\right]
$$

such that each factor $\left(c_{l} z_{i_{1}}+d_{l} z_{i_{2}}\right)^{-1}$ of

$$
f\left(z_{1}, z_{2}\right)=g\left(z_{1}, z_{2}\right) / z_{i_{2}} \prod\left(c_{l} z_{i_{1}}+d_{l} z_{i_{2}}\right), \quad g\left(z_{1}, z_{2}\right) \in \mathbb{C}\left[z_{1}, z_{2}\right]
$$

is expanded in nonnegative integral powers of the variable $z_{i_{2}}$. Clearly, the maps $l_{i_{1} i_{2}}$ are injective. 
Let $V$ be a VOA and $g$ be an automorphism of $V$ of order $N$. Let $M$ be a $g$-twisted $V$-module. Set

$$
M^{\prime}=\prod_{n \in \mathbb{Q}} M_{n}^{*},
$$

the direct sum of the dual spaces of the homogeneous subspaces $M_{n}$ of $M$. Denote by $\langle\cdot, \cdot\rangle$ the natural pairing between $M$ and $M^{\prime}$. Note that for $w^{\prime} \in M^{\prime}, w \in M$ and $v \in V^{j}(j=0, \ldots, N-1)$,

$$
\left\langle w^{\prime}, Y(v, z) w\right\rangle z^{j / N} \in \mathbb{C}\left[z, z^{-1}\right] .
$$

The following proposition can be found in [D2]:

Proposition 2.3. Let $w^{\prime} \in M^{\prime}, w \in M, i, j \in \mathbb{Z}, u \in V^{i}, v \in V^{j}$.

(1) (rationality of product) The element

$$
\left\langle w^{\prime}, Y\left(u, z_{1}\right) Y\left(v, z_{2}\right) w\right\rangle z_{1}^{i / N} z_{2}^{j / N}
$$

involves only integral powers of $z_{1}$ and $z_{2}$ and lies in the image of the map $l_{12}$ :

$$
\left\langle w^{\prime}, Y\left(u, z_{1}\right) Y\left(v, z_{2}\right) w\right\rangle z_{1}^{i / N} z_{2}^{j / N}=l_{12} f\left(z_{1}, z_{2}\right),
$$

where the (uniquely determined) element $f \in \mathbb{C}\left[z_{1}, z_{2}\right]_{s}$ is of the form

$$
f\left(z_{1}, z_{2}\right)=g\left(z_{1}, z_{2}\right) / z_{1}^{r} z_{2}^{s}\left(z_{1}-z_{2}\right)^{t}
$$

for some $g \in \mathbb{C}\left[z_{1}, z_{2}\right]$ and $r, s, t \in \mathbb{Z}$.

(2) (commutativity) We also have

$$
\left\langle w^{\prime}, Y\left(v, z_{2}\right) Y\left(u, z_{1}\right) w\right\rangle z_{1}^{i / N} z_{2}^{j / N}=l_{21} f\left(z_{1}, z_{2}\right),
$$

that is

$$
\text { " } Y\left(u, z_{1}\right) Y\left(v, z_{2}\right) z_{1}^{i / N} z_{2}^{j / N} \text { agrees with } Y\left(v, z_{2}\right) Y\left(u, z_{1}\right) z_{1}^{i / N} z_{2}^{j / N},
$$

as operator-valued rational functions."

\section{Bosonic Realizations of Vertex Operator Algebras and Their Twisted Modules}

In this section, we first recall the construction of VOAs associated with even lattices. We then introduce certain automorphisms of these VOAs and construct the corresponding twisted modules.

We are working in the setting of [FLM2] and [DL1]. Let $L$ be a lattice of rank $l$ for some positive integer $l$ with a symmetric, nondegenerate integral bilinear form $\langle\cdot, \cdot\rangle$ such that $\langle\alpha, \alpha\rangle \in 2 \mathbb{Z}$, that is, $L$ is even. Let $\hat{L}$ be the canonical central extension of $L$ by the cyclic group $\langle \pm 1\rangle: 1 \rightarrow\langle \pm 1\rangle \rightarrow \hat{L} \rightrightarrows L \rightarrow 1$ with the commutator $\operatorname{map} c(\alpha, \beta)=(-1)^{\langle\alpha, \beta\rangle}$ for $\alpha, \beta \in L$. Set $\mathbf{h}=\mathbb{C} \otimes_{\mathbb{Z}} L$ and extend the form $\langle\cdot, \cdot\rangle$ from $L$ to $\mathbf{h}$ by $\mathbb{C}$-linearity. Viewing $\mathbf{h}$ as an abelian algebra, denote by $\hat{\mathbf{h}}=\mathbf{h} \otimes \mathbb{C}\left[t, t^{-1}\right] \oplus \mathbb{C} c$ the corresponding affine Lie algebra. Then $\hat{\mathbf{h}}^{+}=\prod_{n \geqq 0} \mathbf{h} \otimes t^{n}, \hat{\mathbf{h}}^{-}=\prod_{n<0} \mathbf{h} \otimes t^{n}$ are subalgebra of $\hat{\mathbf{h}}$. Consider the induced $\hat{\mathbf{h}}-$ module

$$
M(1)=U(\hat{\mathbf{h}}) \otimes U\left(\hat{\mathbf{h}}^{+} \oplus \mathbb{C} c\right) \mathbb{C} \simeq S\left(\hat{\mathbf{h}}^{-}\right) \quad \text { (linearly) }
$$


$\hat{\mathbf{h}}^{+}$acting trivially on $\mathbb{C}$ and $c$ acting as $1 ; U(\cdot)$ denotes universal enveloping algebra and $S(\cdot)$ denotes symmetric algebra. We shall write $\alpha(n)$ for the operator $\alpha \otimes t^{n}$ for $\alpha \in \mathrm{h}$ and $n \in \mathbb{Z}$.

Form the induced $\hat{L}$-module

$$
\mathbb{C}\{L\}=\mathbb{C}[\hat{L}] \otimes_{\mathbb{C}[ \pm 1]} \mathbb{C} \simeq \mathbb{C}[L] \text { (linearly) },
$$

where $\mathbb{C}(\cdot)$ denotes group algebra and -1 acts on $\mathbb{C}$ as multiplication by -1 . For $a \in \hat{L}$, write $l(a)$ for $a \otimes 1$ in $\mathbb{C}\{L\}$. Then the action of $\hat{L}$ on $\mathbb{C}\{L\}$ is given by: $a \cdot l(b)=\imath(a b)$ and $-1 \cdot l(b)=-l(b)$ for $a, b \in \hat{L}$. Also define an action of $\mathbf{h}$ on $\mathbb{C}\{L\}$ by:

$$
h \cdot l(a)=\langle h, \bar{a}\rangle_{l}(a)
$$

for $h \in \mathbf{h}$. We shall use commuting formal variables $z, z_{0}, z_{1}, z_{2}$. Define an operator $z^{h}$ by

$$
z^{h} \cdot l(a)=z^{\langle h, \bar{a}\rangle} l(a) .
$$

The untwisted space associated with the lattice $L$ is defined to be

$$
V_{L}=M(1) \otimes \mathbb{C} \mathbb{C}\{L\} \simeq S\left(\hat{\mathbf{h}}^{-}\right) \otimes \mathbb{C}[L] \quad \text { (linearly) },
$$

Then $\hat{L}, \hat{\mathbf{h}}, h$ and $z^{h}(h \in \mathbf{h})$ act naturally on $V_{L}$ by acting on either $M(1)$ or $\mathbb{C}\{L\}$ as indicated above.

We shall next define the untwisted vertex operator $Y(v, z)$ for $v \in V_{L}$. For $\alpha \in \mathrm{h}$ set

$$
\alpha(z)=\sum_{n \in \mathbb{Z}} \alpha(n) z^{-n-1}
$$

We use a normal ordering procedure, indicated by open colons, which signify that the enclosed expression is to be reordered if necessary so that all the operators $\alpha(n)$, $(\alpha \in \mathbf{h}, n<0), a \in \hat{L}$ are to be placed to the left of all the operators $\alpha(n), z^{\alpha}(\alpha \in \mathbf{h}, n \geqq 0)$ before the expression is evaluated. For $a \in \hat{L}$, set

$$
Y(l(a), z)=Y(a, z)=: e^{\int\left(\bar{a}(z)-\tilde{a}(0) z^{-1}\right)} a z^{\bar{a}}:,
$$

using an obvious formal integration notation. Let $a \in \hat{L}, \alpha_{1}, \ldots, \alpha_{k} \in \mathbf{h}, n_{1}, \ldots, n_{k} \in$ $\mathbb{Z}\left(n_{i}>0\right)$ and set

$$
\begin{aligned}
v & =\alpha_{1}\left(-n_{1}\right) \ldots \alpha_{k}\left(-n_{k}\right) \otimes l(a) \\
& =\alpha_{1}\left(-n_{1}\right) \ldots \alpha_{k}\left(-n_{k}\right) \cdot l(a) \in V_{L} .
\end{aligned}
$$

We define

$$
Y(v, z)=:\left(\frac{1}{\left(n_{1}-1\right) !}\left(\frac{d}{d z}\right)^{n_{1}-1} \alpha_{1}(z)\right) \ldots\left(\frac{1}{\left(n_{k}-1\right) !}\left(\frac{d}{d z}\right)^{n_{k}-1} \alpha_{k}(z)\right) Y(a, z):
$$

This gives us a well-defined linear map

$$
\begin{aligned}
& V_{L} \rightarrow\left(\operatorname{End} V_{L}\right)[[z]], \\
& v \mapsto Y(v, z)=\sum_{n \in \mathbb{Z}} v_{n} z^{-n-1}, \quad\left(v_{n} \in \text { End } V_{L}\right) .
\end{aligned}
$$

We call $Y(v, z)$ the untwisted vertex operator associated with $v$. 
Let $\left\{\beta_{1}, \ldots, \beta_{l}\right\}$ be an orthonormal basis of $\mathbf{h}$. We set $\omega=\frac{1}{2} \sum_{i=1}^{l} \beta_{i}(-1)^{2} \in V_{L}$. We also set $1=\imath(1) \in V_{L}$. For the next result, see [Bo, FLM2]:

Theorem 3.1. The space $\left(V_{L}, Y, \mathbf{1}, \omega\right)$ is a vertex operator algebra.

Remark 3.2. If $L$ is a root lattice of a Lie algebra $g$ of type $A, D$ or $E$ then $g$ can be identified with $\left(V_{L}\right)_{1}=\left\{v \in V_{L} \mid L(0) v=v\right\}$ (which is a Lie algebra with respect to bracket $[u, v]=u_{0} v$ ) by sending $x \in \mathfrak{g}$ to $x(-1) l(1)$.

Recall the affine Lie algebra

$$
\hat{\mathfrak{g}}=\mathfrak{g} \otimes \mathbb{C}\left[t, t^{-1}\right]+\mathbb{C} c,
$$

whose bracket is given by

$$
\left[u \otimes t^{m}, v \otimes t^{n}\right]=[u, v] \otimes t^{m+n}+c\langle u, v\rangle \delta_{m+n, 0}
$$

for $u, v \in \mathfrak{g}$ and $m, n \in \mathbb{Z}$. For $x \in \mathfrak{g}$, set

$$
x(z)=\sum_{n \in \mathbb{Z}}\left(x \otimes t^{n}\right) z^{-n-1} .
$$

Then the map $x(z) \mapsto Y(x(-1) l(1), z)$ and $c \mapsto 1$ provides an irreducible representation for the affine Lie algebra $\hat{\mathfrak{g}}$ on $V_{L}$ and $V_{L} \simeq L\left(\Lambda_{0}\right)$ is called the fundamental representation (see [DL1]).

Set $\mathbf{h}_{\mathbb{Q}}=\mathbb{Q} \otimes_{\mathbb{Z}} L \subset \mathbf{h}$. Recall that the dual lattice $L^{\circ}$ of $L$ is defined to be $L^{\circ}=\{\beta \in \mathbf{h} \mid\langle\beta, L\rangle \subset \mathbb{Z}\} \subset \mathbf{h}_{\mathbb{Q}}$. Let $\alpha \in \mathbf{h}_{\mathbb{Q}}$. Then there exists a smallest positive integer $N$ such that $\langle\alpha, L\rangle \subset \frac{1}{N} \mathbb{Z}$. We define an element $g_{\alpha} \in G L\left(V_{L}\right)$ determined by

$$
g_{\alpha}(u \otimes l(a))=e^{2 \pi i\langle\alpha, \bar{a}\rangle}(u \otimes l(a))
$$

for $u \in S\left(\hat{\mathbf{h}}^{-}\right)$and $a \in \hat{L}$. We have the following relations:

$$
\begin{gathered}
g_{\alpha} \beta(n) g_{\alpha}^{-1}=\beta(n) \quad \text { for } \beta \in \mathbf{h}, n \in \mathbb{Z}, \\
g_{\alpha} z^{\beta} g_{\alpha}^{-1}=z^{\beta} \quad \text { for } \beta \in L, \\
g_{\alpha} a g_{\alpha}^{-1}=e^{2 \pi i\langle\alpha, \bar{a}\rangle} a \text { for } a \in \hat{L} .
\end{gathered}
$$

Proposition 3.3. (1) $g_{\alpha}$ is an automorphism of the vertex operator algebra $V_{L}$ of order $N$.

(2) $g_{\alpha}=g_{\beta}$ for $\alpha, \beta \in \mathbf{h}_{\mathbb{Q}}$ if and only if $\alpha-\beta \in L^{\circ}$. Thus $\mathbf{h}_{\mathbb{Q}} / L^{\circ}$ can be regarded as a subgroup of the autrmorphism group of $V_{L}$.

(3) If $g$ is an automorphism of $V_{L}$ such that $g(u \otimes l(a))=\eta_{a}(u \otimes l(a))$, where $\eta_{\alpha}$ is a root of unity depending only on $\bar{a}$ for all $u \in S\left(\hat{\mathbf{h}}^{-}\right)$and $a \in \hat{L}$, then there exists $a \in \mathbf{h}_{\mathbb{Q}}$ so that $g=g_{\alpha}$.

Proof. The first two assertions follow from the definition of vertex operators (3.6), (3.7), (3.9) and the relations (3.14)-(3.16). The third assertion is true because $L$ is a finite-generated free abelian group.

Let $\beta \in \mathbf{h}_{\mathbb{Q}}$ such that $\alpha-\beta \in L^{\circ}$. Let $V_{L+\beta}$ be $V_{L}$ as vector spaces. We shall define an action of $V_{L}$ on $V_{L+\beta}$ by using so-called "shifted vertex operators" introduced in [L] (also see [T]). The actions of $\hat{L}$ and $h(n)$ for $h \in \mathbf{h}$ and $0 \neq n \in \mathbb{Z}$ are the same as before, but we define new actions of $h=h(0)$ and $z^{h}$ as follows:

$$
h \cdot u \otimes \imath(a)=\langle h, \bar{a}+\beta\rangle u \otimes \imath(a), \quad z^{h} \cdot u \otimes \imath(a)=z^{\langle h, \bar{a}+\beta\rangle} u \otimes \imath(a)
$$


for $u \in M(1)$ and $a \in \hat{L}$. Denote by $Y_{\beta}(u, z)$ the corresponding operator for $u \in V_{L}$ and call it a shifted vertex operator. Then we have a linear map:

$$
\begin{aligned}
V_{L} & \rightarrow\left(\text { End } V_{L+\beta}\right)\left[\left[z^{1 / N}, z^{-1 / N}\right]\right], \\
v & \mapsto Y_{\beta}(v, z)=\sum_{n \in \frac{1}{N} \mathbb{Z}} v_{n} z^{-n-1}\left(v_{n} \in \operatorname{End} V_{L+\beta}\right) .
\end{aligned}
$$

Let $L^{\circ}=\cup_{i \in L^{\circ} / L}\left(L+\lambda_{i}\right)$ be a coset decomposition such that $\lambda_{0}=0$.

Theorem 3.4. Let $\alpha$ and $\beta$ be as before. Then

(1) The space $\left(V_{L+\beta}, Y_{\beta}\right)$ is an irreducible $g_{\alpha}$-twisted $V_{L}$-module.

(2) The spaces $\left(V_{L+\lambda_{i}-\alpha}, Y_{\lambda_{i}-\alpha}\right)$ for $i \in L^{\circ} / L$ are the unique irreducible $g_{\alpha}$-twisted $V_{L}$-modules up to isomorphism.

Proof. For (1) it is sufficient to prove the relations (2.14) and (2.18). For $k \in \mathbb{Z}$ the eigenspace of $g_{\alpha}$ with eigenvalue $\eta^{k}$ is

$$
V_{L}^{k}=\mathbb{C}-\operatorname{span}\left\{u \otimes \imath(a) \mid u \in S\left(\hat{\mathbf{h}}^{-}\right), a \in \hat{L} \text { such that }\langle\alpha, \bar{a}\rangle \equiv \frac{k}{N} \bmod \mathbb{Z}\right\} .
$$

Thus $Y_{\lambda_{i}-\alpha}(v, z)$ has the expansion (2.14) for $v \in V_{L}^{k}$. Let $u=u^{*} \otimes \imath(a) \in V_{L}^{k}, v=v^{*}$ $\otimes l(b) \in V_{L}$. Using the proof of Theorem 8.8.22 of [FLM2] or the proof of Theorem 5.1 of [DL1], we obtain the desired identity (2.18). The proof of the second assertion in case $\alpha=0$ is given in [D1], and the proof for arbitrary $\alpha$ is similar. We refer the reader to $[D 1]$ for more details.

Next we recall some definitions and results about twisted affine Lie algebras (see $[\mathrm{K}$ and $\mathrm{LW}]$ ). Let $\mathrm{g}$ be a finite-dimensional Lie algebra with a nondegenerate invariant bilinear form $\langle\cdot, \cdot\rangle$ and $g$ be an automorphism of $\mathfrak{g}$ of order $N$. Set $\mathfrak{g}_{k}=\left\{x \in \mathfrak{g} \mid g x=\eta^{k} x\right\}$ for $k \in \mathbb{Z}$. Then $\mathfrak{g}=\mathfrak{g}_{1} \oplus \cdots \oplus \mathfrak{g}_{N}$. For $x \in \mathfrak{g}$, write $x_{(k)}$ for the component of $x$ in $\mathfrak{g}_{k}$. The $g$-twisted affine Lie algebra is defined to be

$$
\hat{\mathrm{g}}[g]=\sum_{n \in \mathbb{Z}} \mathfrak{g}_{n} \otimes t^{n / N} \oplus \mathbb{C} c
$$

with bracket

$$
[x(m), y(n)]=[x, y](m+n)+m\langle x, y\rangle \delta_{m+n, 0} c, \quad[c, \hat{\mathfrak{g}}[g]]=0 .
$$

where $x, y \in \mathfrak{g}, m, n \in \frac{1}{N} \mathbb{Z}, x(m)=x_{(m N)} \otimes t^{m}, y(n)=y_{(n N)} \otimes t^{n}$. If $\mathfrak{g}$ is semi-simple and $g$ is an inner automorphism of $\mathfrak{g}$ then $\hat{\mathrm{g}}[g]$ is isomorphic to $\hat{\mathrm{g}}$. Set

$$
x(z)=\sum_{n \in \frac{1}{N} \mathbb{Z}} x(n) z^{-n-1} .
$$

The following lemma can be verified by a straightforward calculation:

Lemma 3.5. For $x \in \mathfrak{g}_{k}, y \in \mathfrak{g}$,

$\left[x\left(z_{1}\right), y\left(z_{2}\right)\right]=[x, y]\left(z_{2}\right) z_{2}^{-1}\left(\frac{z_{1}}{z_{2}}\right)^{-k / N} \delta\left(\frac{z_{1}}{z_{2}}\right)-\langle x, y\rangle c z_{2}^{-1} \frac{\partial}{\partial z_{1}}\left(\left(\frac{z_{1}}{z_{2}}\right)^{-k / N} \delta\left(\frac{z_{1}}{z_{2}}\right)\right)$.

Remark 3.6. Let $\mathrm{g}$ be as in Remark 3.2. Then $g_{\alpha}$ is an automorphism of the Lie algebra $\mathfrak{g}$ and the vertex operators $Y_{\lambda_{1}-\alpha}(v, z)$ provide an irreducible representation for $\hat{\mathfrak{g}}\left[g_{\alpha}\right]$ with $x(z)=Y_{\lambda_{z}-\alpha}(x(-1) \imath(1), z)$ and $c=1$. 
Finally we discuss the $q$-graded dimension of $V_{L+\beta}$ for $\beta \in \mathbf{h}_{\mathbb{Q}}$. Let $M=\prod_{n \in \mathbb{Q}} M_{n}$ be a graded module for the Virasoro algebra

$$
\operatorname{Vir}=\mathbb{C}-\operatorname{span}\left\{L_{n}, \mathbf{c} \mid n \in \mathbb{Z}\right\}
$$

such that $\mathbf{c}$ acts as a scalar $c \in \mathbb{Q}$ and $L_{0}(v)=n v$ for $n \in \mathbb{Q}$ and $v \in M_{n}$. The $q$-graded dimension of $M$ is defined to be

$$
\operatorname{ch}_{q} M=\operatorname{tr}\left(q^{L_{0}-c / 24}\right)=q^{-c / 24} \sum_{n \in \mathbb{Q}}\left(\operatorname{dim} M_{n}\right) q^{n} .
$$

Recall that $\omega=\frac{1}{2} \sum_{i=1}^{l} \beta_{i}(-1)^{2} \in V_{L}$. It is well-known that

$$
L(0)=\frac{1}{2} \sum_{i=1}^{l} \beta_{i}(0)^{2}+\sum_{i=1}^{l} \sum_{n>0} \beta_{i}(-n) \beta_{i}(n)
$$

and that

$$
\left[L(0), v_{n}\right]=-n v_{n}
$$

for $v \in\left(V_{L}\right)_{1}$ and $n \in \mathbb{Q}$. In particular,

$$
[L(0), \alpha(n)]=-n \alpha(n)
$$

for $\alpha \in \mathbf{h}$. Then from (3.17) we see that for $v=\alpha_{1}\left(-n_{1}\right) \ldots \alpha_{k}\left(-n_{k}\right) \otimes \imath(a) \in V_{L+\beta}$ (cf. (3.8)),

$$
L(0) v=\left(n_{1}+\cdots n_{k}+\frac{1}{2}\langle\bar{a}+\beta, \bar{a}+\beta\rangle\right) v
$$

Set

$$
\eta(q)=q^{1 / 24} \prod_{n>0}\left(1-q^{n}\right), \quad \theta_{L+\beta}(q)=\sum_{\alpha \in L+\beta} q^{\langle\alpha, \alpha\rangle / 2} .
$$

Then from (3.29) we have the following proposition:

Proposition 3.7. The graded-dimension of $V_{L+\beta}$ is $\operatorname{ch}_{q} V_{L+\beta}=\frac{\theta_{L+\beta}(q)}{\eta(q)^{l}}$.

\section{Fermionic Construction of Vertex Operator Algebras and Modules}

In this section, we consider certain automorphisms of finite order of the Lie algebra of type $D_{l}$ and construct the corresponding twisted modules both for $D_{l}^{(1)}$ and for a certain related VOA.

First we review the fermionic construction of the Lie algebra of type $D_{l}$ (see [FFR] for example). Let $(\cdot, \cdot)$ be a nondegenerate symmetric bilinear form on $A \simeq \mathbb{C}^{2 l}$, with $A=A^{+} \oplus A^{-}$a polarization into maximal isotropic subspaces. Then the Clifford algebra $C(A)$ is the associative algebra with 1 , generators $A$ and relations $a b+b a=(a, b) 1$ for $a, b \in A$. Setting $: a b:=\frac{1}{2}(a b-b a)$, the span of all such elements in $C(A)$ is closed under brackets and forms a Lie algebra $g$ of type $D_{l}$. If $x=: a_{1} a_{2}:, y=: b_{1} b_{2}$ : then

$$
[x, y]=\left(a_{1}, b_{2}\right): a_{2} b_{1}:+\left(a_{2}, b_{1}\right): a_{1} b_{2}:-\left(a_{1}, b_{1}\right): a_{2} b_{2}:-\left(a_{2}, b_{2}\right): a_{1} b_{1}:
$$

and the invariant form is

$$
(x, y)=\left(a_{1}, b_{2}\right)\left(a_{2}, b_{1}\right)-\left(a_{1}, b_{1}\right)\left(a_{2}, b_{2}\right) .
$$


For $g \in G L\left(A^{+}\right)=G L(l)$ we have an obvious action of $g$ on $A^{-}$(regarded as the dual space of $\left.A^{+}\right)$by $\left(g^{t}\right)^{-1}$. Then $g \mapsto\left(g,\left(g^{t}\right)^{-1}\right)$ is a representation of $G L\left(A^{+}\right)$ which preserves the bilinear form $(\cdot, \cdot)$. Thus $G L(l)$ is embedded into $S O(2 l)$ and acts on $C(A)$ as algebra automorphisms, and we always take $G L(l)$ to act on $A$ and $C(A)$ in this manner. Let $G$ be a finite subgroup of $G L(l)$. For $g \in G$ of order $N$, let $\left\{a_{\left.1, g, \ldots, a_{l, g}\right\}}\right.$ be a basis of $A^{+}$such that $\eta=e^{2 \pi i / N}$ and

$$
g a_{i, g}=\eta^{n_{1, g}} a_{l, g}
$$

where $n_{i, g} \in \mathbb{Z}$ for $1 \leqq i \leqq l$; let $\left\{a_{i, g}^{*}, \ldots, a_{l, g}^{*}\right)$ be a dual basis of $A^{-}$so that $\left(a_{i, g}\right.$, $\left.a_{j, g}^{*}\right)=\delta_{i, j}$. Then

$$
g a_{i, g}^{*}=\eta^{-n_{i, g}} a_{i, g}^{*}
$$

and $\left\{h_{i, g}=: a_{i, g} a_{i, g}^{*}: \mid 1 \leqq i \leqq l\right\}$ is an orthonormal basis of a Cartan subalgebra $\mathfrak{h}_{g}$ of $\mathfrak{g}$. By identifying $\mathfrak{h}_{g}$ with $\mathfrak{h}_{g}^{*}$ via $(\cdot, \cdot)$ we may choose

$$
\left\{\alpha_{1, g}=h_{1, g}-h_{2, g}, \ldots, \alpha_{l-1, g}=h_{l-1, g}-h_{l, g}, \alpha_{l, g}=h_{l-1, g}+h_{l, g}\right\}
$$

to be a fundamental system of roots. Then $\Delta_{+}=\left\{h_{i, g} \pm h_{j, g} \mid i<j\right\}$ is the set of positive roots, and

$$
L_{g}=\mathbb{Z}-\operatorname{span}\left\{h_{i, g} \pm h_{j, g} \mid 1 \leqq i, j \leqq l\right\} \subset \mathfrak{g}
$$

is the corresponding root lattice. The weight lattice (or dual lattice) is

$$
L_{g}^{\circ}=\left\{x_{1} h_{1, g}+\cdots+x_{l} h_{l, g} \mid x_{i} \in \frac{1}{2} \mathbb{Z} \text { and } \sum x_{i} \in \mathbb{Z}\right\} .
$$

The sublattice

$$
\Gamma_{l, g}=\mathbb{Z}-\operatorname{span}\left\{L_{g}, \sum_{k=1}^{l} \frac{1}{2} h_{k, g}\right\}
$$

of $L_{g}^{\circ}$ is called the spin lattice and is a self dual even lattice if $8 \mid l$ (see [S]). For convenience, we shall omit the index $g$ if $g=1$. For example, we shall write $a_{i}=a_{i, 1}, a_{i}^{*}=a_{i, 1}^{*}$. The next result is easy.

Lemma 4.1. (1) Let $g \in G L(l)$. Then $g(: a b:)=: g(a) g(b):$ for $a, b \in A$ defines an automorphism of $\mathrm{g}$.

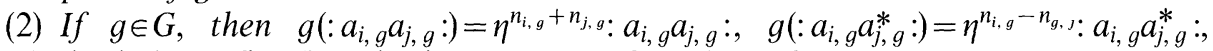
$g\left(: a_{i, g}^{*} a_{j, g}^{*}:\right)=\eta^{-n_{i, g}-n_{g, j}: a_{i, g}^{*}} a_{j, g}^{*}:$ In particular, $\left.g\right|_{\mathfrak{h}_{g}}=i d$.

(3) The vector $h_{1, g}+\cdots+h_{l, g}=h_{1}+\cdots+h_{l}$ is independent of $g$.

Let $g \in G$ have order $N$. Set

$$
A_{k, g}^{ \pm}=\left\{a \in A^{ \pm} \mid g a=\eta^{k} a\right\}, \quad A_{k, g}=A_{k, g}^{+}+A_{k, g}^{-}
$$

for $k \in \mathbb{Z}$. Then $A=\bigoplus_{k \in \mathbb{Z} / N \mathbb{Z}} A_{k, g}$. For $a \in A$ write $a_{(k)}$ for the component of $a$ in $A_{k, g}$ We also set

$$
A\left(\mathbb{Z}+\frac{1}{2}, g\right)=\sum_{n \in \mathbb{Z}} A_{n, g} \otimes t^{n / N-1 / 2}, \quad A(\mathbb{Z}, g)=\sum_{n \in \mathbb{Z}} A_{n, g} \otimes t^{n / N} .
$$

For $a \in A$ and $n \in \frac{1}{N} \mathbb{Z}$, denote $a_{(n N)} \otimes t^{n-1 / 2}$ by $a(n-1 / 2)$ in $A\left(\mathbb{Z}+\frac{1}{2}, g\right)$ and $a_{(n N)} \otimes t^{n}$ by $a(n)$ in $A(\mathbb{Z}, g)$. Then $A(Z, g)$ for $Z=\mathbb{Z}+\frac{1}{2}$ or $\mathbb{Z}$ has a $\mathbb{Q}$-gradations (weight gradation) given by:

$$
\text { wt } a(n)=-n \text {. }
$$


Extend the form $(\cdot, \cdot)$ to $A(Z, g)$ by

$$
(a(m), b(n))=(a, b) \delta_{m+n, 0}
$$

for $a, b \in A, m, n \in \frac{1}{N} \mathbb{Z}+\frac{1}{2}$ or $\frac{1}{N} \mathbb{Z}$. Set

$$
A\left(\mathbb{Z}+\frac{1}{2}, g\right)^{ \pm}=A_{\bar{N} / 2, g}^{ \pm}+\sum_{n-\frac{1}{2} \gtrless 0} A_{n, g} \otimes t^{n-1 / 2}
$$

(where $A_{\bar{N} / 2, g}^{ \pm}$is understood to be 0 if $N$ is odd) and

$$
A(\mathbb{Z}, g)^{ \pm}=A_{0, g}^{ \pm}+\sum_{n \gtrless 0} A_{n, g} \otimes t^{n}
$$

Then $A(Z, g)=A^{+}(Z, g) \oplus A^{-}(Z, g)$ is a polarization. As before we denote by $C(A(Z, g))$ the Clifford algebra with 1 , generators $A(Z, g)$ and relations

$$
a(m) b(n)+b(n) a(m)=\delta_{m+n, 0}(a, b) .
$$

Let $C^{+}(A(Z, g))$ be the subalgebra of $C(A(Z, g))$ generated by $A^{+}(Z, g)$, and form the induced module

$$
C M(Z, g)=C(A(Z, g)) \otimes_{C^{+}(A(Z, g))} \mathbb{C} \mathbf{1}_{Z, g} \simeq \Lambda\left(A^{-}(Z, g)\right),
$$

where $\mathbb{C} \mathbf{1}_{Z, g}$ is a one-dimensional $C^{+}(A(Z, g))$-module such that $A^{+}(Z, g)$ annihilates $\mathbf{1}_{Z, g}$ and 1 acts as the identity. The symbol $\Lambda(\cdot)$ denotes the exterior algebra. Then $C M(Z, g)$ is an irreducible module for $C(A(Z, g))$ and it decomposes into even and odd parity subspaces

$$
C M(Z, g)=C M^{0}(Z, g) \oplus C M^{1}(Z, g),
$$

where there are linear isomorphisms.

$$
C M^{0}(Z, g) \simeq \Lambda^{\text {even }}\left(A^{-}(Z, g)\right), \quad C M^{1}(Z, g) \simeq \Lambda^{\text {odd }}\left(A^{-}(Z, g)\right) .
$$

Remark 4.2. We endow $C M(Z, g)$ with a $\mathbb{Q}$-gradation as follows:

$$
\mathrm{wt1}_{Z, g}=c_{Z, g}, \quad \operatorname{wt}(a(n) u)=\mathrm{wt} a(n)+\mathrm{wt} u
$$

for $a \in A, n \in \frac{1}{2 N} \mathbb{Z}$ and $u \in C M(Z, g)$, where

$$
c_{Z, g}= \begin{cases}\frac{1}{2} \sum_{0 \leqq k<\frac{N}{2}}\left(\frac{k}{N}\right)^{2} \operatorname{dim} A_{k, g}^{+}+\sum_{\frac{N}{2} \leqq k<N}\left(1-\frac{k}{N}\right)^{2} \operatorname{dim} A_{k, g}^{+} & \text {if } Z=\mathbb{Z}+\frac{1}{2} \\ \frac{1}{2} \sum_{0 \leqq k<N}\left(\frac{k}{N}-\frac{1}{2}\right)^{2} \operatorname{dim} A_{k, g}^{+} & \text {if } Z=\mathbb{Z} .\end{cases}
$$

The reason for choosing this gradation will be explained later.

Remark 4.3. Let $g \in G$. If $h \in C_{G}(g)$ then $h$ preserves each $A_{k . g}^{ \pm}$and thus acts on $A(Z, g)$ by

$$
h a(n)=(h a)(n) .
$$

Note that this action preserves the $\mathbb{Q}$-gradation of $A(Z, g)$ and the form (4.11). Then there is a unique action of $C_{G}(h)$ on $C M(Z, g)$ such that

$$
h \cdot \mathbf{1}_{Z, g}=\mathbf{1}_{Z, g}, \quad h a(n) h^{-1}=(h a)(n) .
$$


Evidently $h$ preserves the $\mathbb{Q}$-gradation of $C M(Z, g)$, whence, $C M(Z, g)$ is a $\mathbb{Q}$ graded $C_{G}(g)$-module.

For $a \in A$, set

$$
a(z)=\sum_{n \in \frac{1}{N} \mathbb{Z}} a\left(n-\frac{1}{2}\right) z^{-n}
$$

on $C M\left(\frac{1}{2}+\mathbb{Z}, g\right)$ and

$$
a(z)=\sum_{n \in \frac{1}{N} \mathbb{Z}} a(n) z^{-n-1 / 2}
$$

on $C M(\mathbb{Z}, g)$. Define a normal ordering: :

$$
: a(m) b(n):= \begin{cases}a(m) b(n) & \text { if } m<n \\ \frac{1}{2}(a(m) b(n)-b(n) a(m)) & \text { if } m=n \\ -b(n) a(m) & \text { if } m>n .\end{cases}
$$

Then $: a(m) b(n):=-: b(n) a(m):$. Define linear functions $\psi_{Z, g}: \mathfrak{g} \rightarrow \mathbb{C}$ by

$$
\psi_{Z, g}(u)= \begin{cases}(k / N-1 / 2)(a, b) & \text { if } k \neq 0 \\ 0 & \text { if } k=0\end{cases}
$$

and

$$
\psi_{\mathbb{Z}+\frac{1}{2}, g}(u)= \begin{cases}k / N(a, b) & \text { if } k / N<1 / 2 \\ 0 & \text { if } k / N=1 / 2, \\ (k / N-1)(a, b) & \text { if } k / N>1 / 2\end{cases}
$$

where $u=: a b: \in \mathfrak{g}$ with $a \in A_{k, g}$ and $b \in A_{m, g}$ and $0 \leqq k, m \leqq N$.

Lemma 4.4. The functions $\psi$ are well-defined.

Proof. It is enough to show that $\psi(: a b:)=\psi(-: b a:)$. If $(a, b)=0$, this is clear, so without loss of generality we can assume that $a \in A_{k, g}^{+}$and $b=A_{N-k, g}^{-}$. If $k=0$ then $\psi_{Z, g}(: a b:)=\psi_{Z, g}(-: b a:)=0$. If $k \neq 0$ then $\psi_{Z, g}(: a b:)=(k / N-1 / 2)(a, b)$ and $\psi_{Z, g}(-: b a:)=-((N-k) / N-1 / 2)(b, a)$, as desired. Similarly for $\psi_{\mathbb{Z}+\frac{1}{2}}$.

For $u=: a b: \in \mathfrak{g}$ set

$$
Y(u, z)=: a(z) b(z):-\psi_{z}(u) z^{-1}
$$

on $C M(Z, g)$. Then $Y(u, z)$ is well-defined and for $r=0,1, C M(Z, g)^{r}$ is invariant under the component operators of $Y(u, z)$. Next we show that the operators $Y(u, z)$ provide a representation of $\hat{\mathrm{g}}[g]$ on $C M(Z, g)$. Since the arguments for $Z=\mathbb{Z}$ and $Z=\frac{1}{2}+\mathbb{Z}$ are essentially the same we only give the proof for the former case, so from now we assume that $Z=\mathbb{Z}$.

For $a, b \in A$ and $m, n \in \frac{1}{N} \mathbb{Z}$ define the contraction

$$
\underbrace{a(m) b(n)}=a(m) b(n)-: a(m) b(n): .
$$

We also have an analogous notation $a\left(z_{1}\right) b\left(z_{2}\right)$. Recalling (4.22), set

$$
a^{ \pm}(z)=\sum_{n \gtrless 0} a(n) z^{-n-1 / 2}+\frac{1}{2} a(0) z^{-1 / 2} .
$$


For $0 \leqq k \leqq N-1$ define a function

$$
f_{k}\left(z_{1}, z_{2}\right)= \begin{cases}z_{1}^{1 / 2-k / N} z_{2}^{k / N-1 / 2} & \text { if } k \neq 0 \\ \frac{1}{2}\left(z_{1}^{1 / 2} z_{2}^{-1 / 2}+z_{1}^{-1 / 2} z_{2}^{1 / 2}\right) & \text { if } k=0 .\end{cases}
$$

The following lemma is straightforward.

Lemma 4.5. Let $a \in A_{k}$ and $b \in A$. Then

$$
\underbrace{a\left(z_{1}\right) b\left(z_{2}\right)}=\left[a^{+}\left(z_{1}\right), b\left(z_{2}\right)\right]_{+}=a^{+}\left(z_{1}\right) b\left(z_{2}\right)+b\left(z_{2}\right) a^{+}\left(z_{1}\right)=(a, b) \frac{f_{k}\left(z_{1}, z_{2}\right)}{z_{1}-z_{2}}
$$

and

$$
\underbrace{b\left(z_{2}\right) a\left(z_{1}\right)}=\left[b^{+}\left(z_{1}\right), a\left(z_{2}\right)\right]_{+}=(a, b) \frac{f_{k}\left(z_{1}, z_{2}\right)}{z_{2}-z_{1}} \text {. }
$$

In general, for $a_{1}, \ldots, a_{k} \in A$ and $n_{1}, \ldots, n_{k} \in \frac{1}{2 N} \mathbb{Z}$ define

$$
: a_{1}\left(n_{1}\right) \ldots a_{k}\left(n_{k}\right):=a_{1}\left(n_{1}\right) \ldots a_{k}\left(n_{k}\right)
$$

if $n_{1} \leqq \ldots \leqq n_{k}$ and $n_{i} \neq 0$ and

$$
: a_{\sigma(1)}\left(n_{\sigma(1)}\right) \ldots a_{\sigma(k)}\left(n_{\sigma(k)}\right):=(-1)^{|\sigma|}: a_{1}\left(n_{1}\right) \ldots a_{k}\left(n_{k}\right):
$$

for $\sigma \in S_{k}$. We also define

$$
: a_{1}(0) \ldots a_{k}(0):=\frac{1}{k} \sum_{\sigma \in S_{k}} a_{\sigma(1)}(0) \ldots a_{\sigma(k)}(0) .
$$

If $n_{1} \leqq \ldots \leqq n_{i}<0=n_{i+1}=\ldots=n_{j}<n_{j+1} \leqq \ldots \leqq n_{k}$, define

$$
: a_{1}\left(n_{1}\right) \ldots a_{k}\left(n_{k}\right):=a_{1}\left(n_{1}\right) \ldots a_{i}\left(n_{i}\right): a_{i+1}(0) \ldots a_{j}(0): a_{j+1}\left(n_{j+1}\right) \ldots a_{k}\left(n_{k}\right) \text {. }
$$

The following lemma is a direct consequence of the definition of normal ordering and the contraction, and is sometimes referred to as the Wick theorem [W].

Lemma 4.6. Let $a_{1}, a_{2}, b_{1}, b_{2} \in A$ and $m_{1}, m_{2}, n_{1}, n_{2} \in \frac{1}{N} \mathbb{Z}$. Then

$$
\begin{aligned}
: a_{1}\left(m_{1}\right) a_{2}\left(m_{2}\right):: b_{1}\left(n_{1}\right) b_{2}\left(n_{2}\right):=: a_{1}\left(m_{1}\right) a_{2}\left(m_{2}\right) b_{1}\left(n_{1}\right) b_{2}\left(n_{2}\right): & \\
& +\underbrace{a_{1}\left(m_{1}\right) b_{2}\left(n_{2}\right)}: a_{2}\left(m_{2}\right) b_{1}\left(n_{1}\right):+\underbrace{a_{2}\left(m_{2}\right) b_{1}\left(n_{1}\right)}: a_{1}\left(m_{1}\right) b_{2}\left(n_{2}\right): \\
& -\underbrace{a_{1}\left(m_{1}\right) b_{1}\left(n_{1}\right)}: a_{2}\left(m_{2}\right) b_{2}\left(n_{2}\right):-\underbrace{a_{2}\left(m_{2}\right) b_{2}\left(n_{2}\right)}: a_{1}\left(m_{1}\right) b_{1}\left(n_{1}\right): \\
& +\underbrace{a_{1}\left(m_{1}\right) b_{2}\left(n_{2}\right)} \underbrace{a_{2}\left(m_{2}\right) b_{1}\left(n_{1}\right)}-\underbrace{a_{1}\left(m_{1}\right) b_{1}\left(n_{1}\right)} \underbrace{a_{2}\left(m_{2}\right) b_{2}\left(n_{2}\right)} .
\end{aligned}
$$

From Lemmas 4.5 and 4.6 we have:

Lemma 4.7. Let $a_{1} \in A_{j, g}, a_{2} \in A_{k, g}$ with $0 \leqq j, k \leqq N-1$ and $b_{1}, b_{2} \in A$. Then

(1) $: a_{1}\left(z_{1}\right) a_{2}\left(z_{1}\right):: b_{1}\left(z_{2}\right) b_{2}\left(z_{2}\right):=: a_{1}\left(z_{1}\right) a_{2}\left(z_{1}\right) b_{1}\left(z_{2}\right) b_{2}\left(z_{2}\right)$ :

$$
\begin{aligned}
& +\left(a_{1}, b_{2}\right): a_{2}\left(z_{1}\right) b_{1}\left(z_{2}\right): \frac{f_{j}\left(z_{1}, z_{2}\right)}{z_{1}-z_{2}} \\
& +\left(a_{2}, b_{1}\right): a_{1}\left(z_{1}\right) b_{2}\left(z_{2}\right): \frac{f_{k}\left(z_{1}, z_{2}\right)}{z_{1}-z_{2}}
\end{aligned}
$$




$$
\begin{aligned}
& -: a_{2}\left(z_{1}\right) b_{2}\left(z_{2}\right):\left(a_{1}, b_{1}\right) \frac{f_{j}\left(z_{1}, z_{2}\right)}{z_{1}-z_{2}} \\
& -\left(a_{2}, b_{2}\right): a_{1}\left(z_{1}\right) b_{1}\left(z_{2}\right): \frac{f_{k}\left(z_{1}, z_{2}\right)}{z_{1}-z_{2}} \\
& +\left(a_{1}, b_{2}\right)\left(a_{2}, b_{1}\right) \frac{f_{j}\left(z_{1}, z_{2}\right) f_{k}\left(z_{1}, z_{2}\right)}{\left(z_{1}-z_{2}\right)^{2}} \\
& -\left(a_{1}, b_{1}\right)\left(a_{2}, b_{2}\right) \frac{f_{j}\left(z_{1}, z_{2}\right) f_{k}\left(z_{1}, z_{2}\right)}{\left(z_{1}-z_{2}\right)^{2}} .
\end{aligned}
$$

(2) $: b_{1}\left(z_{2}\right) b_{2}\left(z_{2}\right):: a_{1}\left(z_{1}\right) a_{2}\left(z_{1}\right):=: a_{1}\left(z_{1}\right) a_{2}\left(z_{1}\right) b_{1}\left(z_{2}\right) b_{2}\left(z_{2}\right)$ :

$$
\begin{aligned}
& -\left(a_{1}, b_{2}\right): a_{2}\left(z_{1}\right) b_{1}\left(z_{2}\right): \frac{f_{j}\left(z_{1}, z_{2}\right)}{z_{2}-z_{1}} \\
& -\left(a_{2}, b_{1}\right): a_{1}\left(z_{1}\right) b_{2}\left(z_{2}\right): \frac{f_{k}\left(z_{1}, z_{2}\right)}{z_{2}-z_{1}} \\
& +\left(a_{1}, b_{1}\right): a_{2}\left(z_{1}\right) b_{2}\left(z_{2}\right): \frac{f_{j}\left(z_{1}, z_{2}\right)}{z_{2}-z_{1}} \\
& +\left(a_{2}, b_{2}\right): a_{1}\left(z_{1}\right) b_{1}\left(z_{2}\right): \frac{f_{k}\left(z_{1}, z_{2}\right)}{z_{2}-z_{1}} \\
& +\left(a_{1}, b_{2}\right)\left(a_{2}, b_{1}\right) \frac{f_{j}\left(z_{1}, z_{2}\right) f_{k}\left(z_{1}, z_{2}\right)}{\left(z_{2}-z_{1}\right)^{2}} \\
& -\left(a_{1}, b_{1}\right)\left(a_{2}, b_{2}\right) \frac{f_{j}\left(z_{1}, z_{2}\right) f_{k}\left(z_{1}, z_{2}\right)}{\left(z_{1}-z_{2}\right)^{2}}
\end{aligned}
$$

Set $u=: a_{1} b_{1}:, v=: b_{1} b_{2}: \in \mathfrak{g}$. Using (4.27) we have

$$
\begin{aligned}
{\left[Y\left(u, z_{1}\right), Y\left(v, z_{2}\right)\right]=} & \left(a_{1}, b_{2}\right): a_{2}\left(z_{1}\right) b_{1}\left(z_{2}\right): f_{j}\left(z_{1}, z_{2}\right)\left(\frac{1}{z_{1}-z_{2}}+\frac{1}{z_{2}-z_{1}}\right) \\
& +\left(a_{2}, b_{1}\right): a_{1}\left(z_{1}\right) b_{2}\left(z_{2}\right): f_{k}\left(z_{1}, z_{2}\right)\left(\frac{1}{z_{1}-z_{2}}+\frac{1}{z_{2}-z_{1}}\right) \\
& -\left(a_{1}, b_{1}\right): a_{2}\left(z_{1}\right) b_{2}\left(z_{2}\right): f_{j}\left(z_{1}, z_{2}\right)\left(\frac{1}{z_{1}-z_{2}}+\frac{1}{z_{2}-z_{1}}\right) \\
& -\left(a_{2}, b_{2}\right): a_{1}\left(z_{1}\right) b_{1}\left(z_{2}\right): f_{k}\left(z_{1}, z_{2}\right)\left(\frac{1}{z_{1}-z_{2}}+\frac{1}{z_{2}-z_{1}}\right) \\
& +\left(a_{1}, b_{2}\right)\left(a_{2}, b_{1}\right) f_{j}\left(z_{1}, z_{2}\right) f_{k}\left(z_{1}, z_{2}\right)\left(\frac{1}{\left(z_{1}-z_{2}\right)^{2}}-\frac{1}{\left(z_{2}-z_{1}\right)^{2}}\right) \\
& -\left(a_{1}, b_{1}\right)\left(a_{2}, b_{2}\right) f_{j}\left(z_{1}, z_{2}\right) f_{k}\left(z_{1}, z_{2}\right)\left(\frac{1}{\left(z_{1}-z_{2}\right)^{2}}-\frac{1}{\left(z_{2}-z_{1}\right)^{2}}\right) .
\end{aligned}
$$


The following relations about $\delta$-functions are well known (see, for example, [FLM2]):

$$
\begin{gathered}
\left(z_{1}-z_{2}\right)^{-1}+\left(z_{2}-z_{1}\right)^{-1}=z_{2}^{-1} \delta\left(z_{1} / z_{2}\right) \\
\left(z_{1}-z_{2}\right)^{-2}-\left(z_{2}-z_{1}\right)^{-2}=-z_{2}^{-1} \frac{\partial}{\partial z_{1}} \delta\left(z_{1} / z_{2}\right)
\end{gathered}
$$

Thus

$$
\left[Y\left(u, z_{1}\right), Y\left(v, z_{2}\right)\right]=z_{2}^{-1}\left(\frac{z_{1}}{z_{2}}\right)^{-\frac{j+k}{N}} \delta\left(\frac{z_{1}}{z_{2}}\right) Y\left([u, v], z_{2}\right)+(*)
$$

where

$$
\begin{aligned}
(*)= & \left(\frac{z_{1}}{z_{2}}\right)^{-\frac{j+k}{N}} z_{2}^{-2} \delta\left(\frac{z_{1}}{z_{2}}\right)\left(\left(a_{1}, b_{2}\right) \psi_{\mathbb{Z}, g}\left(: a_{2} b_{1}:\right)+\left(a_{2}, b_{1}\right) \psi_{\mathbb{Z}, g}\left(: a_{1} b_{2}:\right)\right. \\
& \left.-\left(a_{1}, b_{1}\right) \psi_{\mathbb{Z}, g}\left(: a_{2} b_{2}:\right)-\left(a_{2}, b_{2}\right) \psi_{\mathbb{Z}, g}\left(: a_{1} b_{1}:\right)\right) \\
& -(u, v) f_{j}\left(z_{1}, z_{2}\right) f_{k}\left(z_{1}, z_{2}\right) z_{2}^{-1} \frac{\partial}{\partial z_{1}} \delta\left(\frac{z_{1}}{z_{2}}\right) .
\end{aligned}
$$

Lemma 4.8. We have

$$
(*)=-(u, v) z_{2}^{-1} \frac{\partial}{\partial z_{1}}\left(\left(\frac{z_{1}}{z_{2}}\right)^{-\frac{j+k}{N}} \delta\left(\frac{z_{1}}{z_{2}}\right)\right),
$$

or equivalently,

$$
\begin{aligned}
{\left[Y\left(u, z_{1}\right), Y\left(v, z_{2}\right)\right]=} & z_{2}^{-1}\left(\frac{z_{1}}{z_{2}}\right)^{-\frac{j+k}{N}} \delta\left(\frac{z_{1}}{z_{2}}\right) Y\left([u, v], z_{2}\right) \\
& -(u, v) z_{2}^{-1} \frac{\partial}{\partial z_{1}}\left(\left(\frac{z_{1}}{z_{2}}\right)^{-\frac{j+k}{N}} \delta\left(\frac{z_{1}}{z_{2}}\right)\right) .
\end{aligned}
$$

Proof. There are three cases: (1) $j \neq 0, k \neq 0,(2) j \neq 0, k=0,(3) j=k=0$. Since the proofs are similar we only deal the first. Then

$$
(*)=(u, v)\left(\frac{z_{1}}{z_{2}}\right)^{-\frac{j+k}{N}} z_{2}^{-2} \delta\left(\frac{z_{1}}{z_{2}}\right)\left(\frac{j+k}{N}-1\right)-(u, v) f_{j}\left(z_{1}, z_{2}\right) f_{k}\left(z_{1}, z_{2}\right) z_{2}^{-1} \frac{\partial}{\partial z_{1}} \delta\left(\frac{z_{1}}{z_{2}}\right),
$$

and

$$
\begin{aligned}
f_{j}\left(z_{1}, z_{2}\right) f_{k}\left(z_{1}, z_{2}\right) z_{2}^{-1} \frac{\partial}{\partial z_{1}} \delta\left(\frac{z_{1}}{z_{2}}\right)= & z_{2}^{-1} \frac{\partial}{\partial z_{1}}\left(\left(\frac{z_{1}}{z_{2}}\right)^{-\frac{j+k}{N}} \delta\left(\frac{z_{1}}{z_{2}}\right)\right) \\
& -z_{2}^{-2}\left(1-\frac{j+k}{N}\right)\left(\frac{z_{1}}{z_{2}}\right)^{-\frac{j+k}{N}} \delta\left(\frac{z_{1}}{z_{2}}\right) .
\end{aligned}
$$

The result follows.

Finally from Lemmas 3.5 and 4.8 we have 
Theorem 4.9. The spaces $C M(Z, g)$ are $\hat{\mathrm{g}}[g]$-modules on which $u(z)$ acts as $Y(u, z)$ $(u \in \mathfrak{g})$ and $c$ acts as 1 .

This theorem in the case $g=1$ can be found in [F, FF and FFR]. Following the arguments in $[\mathrm{F}$ and $\mathrm{FF}]$ we next show that $C M^{0}(Z, g)$ and $C M^{1}(Z, g)$ are irreducible $\hat{g}[g]$-modules. For $u, v \in \mathfrak{g}$ define a new normal ordering

$$
: u(m) v(n):= \begin{cases}u(m) v(n) & \text { if } m<n \\ \frac{1}{2}(u(m) v(n)+v(n) u(m)) & \text { if } m=n \\ v(n) u(m) & \text { if } m>n .\end{cases}
$$

For $1 \leqq i, j \leqq l$, set

$$
x_{i j, g}=: a_{i, g} a_{j, g}:, \quad x_{i j, g}^{*}=: a_{i, g} a_{j, g}^{*}:, \quad x_{i j, g}^{* *}=: a_{i, g}^{*} a_{j, g}^{*}: .
$$

Then $x_{i j, g}$ is a root vector with root $h_{i, g}+h_{j, g}, x_{i j, g}^{* *}$ is a root vector with root $-h_{i, g}-h_{j, g}$, and $x_{i j, g}^{*}$ is a root vector with root $h_{i, g}-h_{j, g}$ for $i \neq j$.

Lemma 4.10. For $1 \leqq i \leqq l$, we have

(1) $h_{i, g}\left(z_{1}\right) h_{i, g}\left(z_{2}\right)=: h_{i, g}\left(z_{1}\right) h_{i, g}\left(z_{2}\right):+\left(z_{1}-z_{2}\right)^{-2}$,

(2) $x_{i i, g}\left(z_{1}\right) x_{i, g}^{* *}\left(z_{2}\right)=: x_{i i, g}\left(z_{1}\right) x_{i, g}^{* *}\left(z_{2}\right):$.

Proof. (1) follows from the commutator relation

$$
\left[h_{i, g}(m), h_{i, g}(n)\right]=m \delta_{m+n .0}
$$

for $m, n \in \mathbb{Z}$. For (2) note that $\left[x_{i i, g}, x_{i i, g}^{* *}\right]=0$ and $\left\langle x_{i i, g}, x_{i i, g}^{* *}\right\rangle=0$. Thus $\left[x_{i i, g}(m)\right.$, $\left.x_{i i, g}^{* *}(n)\right]=0$ and (2) follows.

Set

$$
D_{i, g}(z)=\sum_{m \in \mathbb{Z}} \sum_{n \in \mathbb{Z}}(2 n-m): a_{i, g}\left(n+n_{i, g} / N\right) a_{i, g}^{*}\left(m-n-n_{i, g} / N\right): z^{-m-2} .
$$

Then we have

Lemma 4.11. For $1 \leqq i \leqq l$,

(1) $: h_{i, g}(z) h_{i, g}(z):=-: a_{i, g}(z) a_{i, g}(z) a_{i, g}^{*}(z) a_{i, g}^{*}(z):+D_{i, g}(z)$.

(2) $: x_{i i, g}(z) x_{i i, g}^{* *}(z):=: a_{i, g}(z) a_{i, g}(z) a_{i, g}^{*}(z) a_{i, g}^{*}(z):$.

Proof. Since the proof for $Z=\mathbb{Z}$ and $Z=\mathbb{Z}+\frac{1}{2}$ are similar we only give the details for $Z=\mathbb{Z}$. Firse let $0<n_{i, g}<N$. From Lemma 4.7, we have

$$
\begin{aligned}
: h_{i, g}\left(z_{1}\right) h_{i, g}\left(z_{2}\right):= & -: a_{i, g}\left(z_{1}\right) a_{i, g}\left(z_{2}\right) a_{i, g}^{*}\left(z_{1}\right) a_{i, g}^{*}\left(z_{2}\right): \\
& -: a_{i, g}\left(z_{2}\right) a_{i, g}^{*}\left(z_{1}\right): \frac{f_{n_{i, g}}\left(z_{1}, z_{2}\right)}{z_{1}-z_{2}}+: a_{i, g}\left(z_{1}\right) a_{i, g}^{*}\left(z_{2}\right): \frac{f_{N-n_{i, g}}\left(z_{1}, z_{2}\right)}{z_{1}-z_{2}} \\
& -\left(n_{i, g} / N-1 / 2\right): a_{i, g}\left(z_{2}\right) a_{i, g}^{*}\left(z_{2}\right):-\left(1 / 2-n_{i, g} / N\right): a_{i, g}\left(z_{1}\right) a_{i, g}^{*}\left(z_{1}\right): .
\end{aligned}
$$

Then the result follows by taking the limit as $z_{1} \rightarrow z_{2}$. The case $n_{i, g}=0$ can be treated similarly.

Recall the constant $c_{Z, g}$ from (4.19), and set

$$
L_{i, g}(z)=c_{Z, g} z^{-2}-\frac{1}{2} D_{i, g}(z)-\frac{n_{i, g}}{N} z^{-1} h_{i, g}(z)=\sum_{n \in \mathbb{Z}} L_{i g}(n) z^{-n-2} .
$$


From Lemma 4.11 the component operators $L_{i, g}(n)$ preserve any submodule of $C M(Z, g)$.

This fact will be crucial in proving that each $C M^{r}(Z, g)$ is irreducible. We also set

$$
L_{g}(z)=\sum_{i=1}^{l} L_{i, g}(z)=\sum_{n \in \mathbb{Z}} L_{g}(n) z^{-n-2}
$$

Proposition 4.12. For $1 \leqq i, j \leqq 1, k, m \in \mathbb{Z}, n \in \frac{1}{2 N} \mathbb{Z}, p \in \frac{1}{N} \mathbb{Z}$ and $u \in \mathfrak{g}$ we have:

(1) $\left[L_{i, g}(k), a_{j, g}(n)\right]=-\delta_{i, j}(n+k / 2) a_{j, g}(k+n)$.

(2) $\left[L_{i, g}(k), a_{j, g}^{*}(n)\right]=-\delta_{i, j}(n+k / 2) a_{j, g}^{*}(k+n)$.

(3) $\left[L_{g}(k), u(p)\right]=-p u(k+n)$

(4) $\left[L_{g}(k), L_{g}(m)\right]=(k-m) L_{g}(k+m)+\frac{l}{12}\left(k^{3}-k\right) \delta_{k+m, 0}$.

Proof. Again we only prove the proposition for $Z=\mathbb{Z}$. In this case $n \in \frac{1}{N} \mathbb{Z}$ and

$$
L_{i, g}(k)=\delta_{k, 0} c_{\mathbb{Z}, g}+\sum_{q \in \mathbb{Z}}\left(k / 2-q-n_{i, g} / N\right): a_{i, g}\left(q+n_{i, g} / N\right) a_{i, g}^{*}\left(k-q-n_{i, g} / N\right):
$$

Noting that $\left[: a_{i, g}\left(q+n_{i, g} / N\right) a_{i, g}^{*}\left(k-q-n_{i, g} / N\right):, a_{i, g}(n)\right]=0$ if $q+n_{i, g} / N \neq n+k$, we obtain:

$$
\left[L_{i, g}(k), a_{i, g}(n)\right]=-(n+k / 2)\left[: a_{i, g}(n+k) a_{i, g}^{*}(-n):, a_{i, g}(n)\right]=-(n+k / 2) a_{i, g}(k+n),
$$

as desired. If $i \neq j$, clearly, $L_{i, g}(k)$ and $a_{j, g}(n)$ commute. The proof of (2) is identical. (3) and (4) follow from (1) and (2).

Now we are in a position to prove the following theorem:

Theorem 4.13. The $\hat{\mathfrak{g}}[g]$-modules $C M^{r}(Z, g)$ are irreducible if $l>1$.

Proof. As we have already pointed out, the component operators $L_{i, g}(n)$ preserve each $\hat{\mathfrak{g}}[g]$-submodule of $C M^{r}(Z, g)$. Recall (4.34) and let $i \neq j$. Then $\psi_{\mathbb{Z}, g}\left(x_{i j}^{*}\right)=0$ (see (4.25)) and $Y\left(x_{i j}, z\right)=: a_{i, g}(z) a_{j, g}^{*}(z):($ see (4.27).) From Proposition 4.12 (1) we get

$$
z_{0}^{L_{i, g}(0)} Y\left(x_{i j}, z\right) e^{-L_{i, g}(0) z_{0}}=z_{0}^{n_{i, g} / N}: a_{i, g}\left(z z_{0}\right) a_{j, g}(z):
$$

and its component operators $a_{i, g}(m) a_{j, g}^{*}(n)$ preserve irreducible submodules. Similarly for the operators $a_{i, g}(m) a_{j, g}^{*}(n), a_{i, g}^{*}(m) a_{j, g}^{*}(n)$. It being clear that $C M^{0}(Z, g)$ and $C M^{1}(Z, g)$ are irreducible under these operators, the proof is complete.

Remark 4.14. Theorem 4.13 was proved for $g=1$ in $[F$ and FF]. In [FFR] the action of $\hat{\mathfrak{g}}$ is extended to an action of $C M^{0}\left(\frac{1}{2}+\mathbb{Z}\right)$ :

$$
Y: C M^{0}\left(\frac{1}{2}+\mathbb{Z}\right) \rightarrow(\operatorname{End} C M(Z))\left[\left[z, z^{-1}\right]\right]
$$

such that $\left(C M^{0}\left(\frac{1}{2}+\mathbb{Z}\right), Y, \mathbf{1}_{\frac{1}{2}+\mathbb{Z}}, \omega\right)$ is a simple VOA, where

$$
\omega=L(-2) \mathbf{1}_{\mathbb{Z}+\frac{1}{2}}=\frac{1}{2} \sum_{i=1}^{l}\left(a_{i}^{*}(-3 / 2) a_{i}(-1 / 2)+a_{i}(-3 / 2) a_{i}^{*}(-1 / 2)\right) \mathbf{1}_{\mathbb{Z}+\frac{1}{2}} .
$$

Moreover, the four spaces $C M^{r}(Z)$ are irreducible $C M^{0}\left(\frac{1}{2}+\mathbb{Z}\right)$-modules. 
Remark 4.15. Recall from (4.21) the action of $G$ on $C M(Z)$. Then $g u(z) g^{-1}=(g u)(z)$ for $u \in \mathfrak{g}$. Since $C M^{0}\left(\mathbb{Z}+\frac{1}{2}\right)$ ) is generated by $g$ (regarded as a weight one subspace of $C M^{0}\left(\mathbb{Z}+\frac{1}{2}\right)$ ) in the sense of [FHL] (also see [DL1]), the relation $g Y(u, z) g^{-1}=$ $Y(g u, z)$ holds for arbitrary $u \in C M^{0}\left(\mathbb{Z}+\frac{1}{2}\right)$. That is, $G$ is a group of automorphisms of the VOA $C M^{0}\left(\mathbb{Z}+\frac{1}{2}\right)$.

Finally we justify the weight gradation of $C M(Z, g)$ introduced in Remark 4.2 by using the operator $L_{g}(0)$ to compute the graded-dimension of $\operatorname{ch}_{q} C M^{r}(Z, g)$. From (4.35)-(4.37) we find that

$$
L_{g}(0)=c_{Z, g}-\sum_{i=1}^{l} \sum_{n \in \mathbb{Z}}\left(n+n_{i, g} / N\right): a_{i, g}\left(n+n_{i, g} / N\right) a_{i, g}^{*}\left(-n-n_{i, g} / N\right): .
$$

It turns out that the weight of $\mathbf{1}_{Z, g}$ defined in (4.18) is precisely the $L_{g}(0)$-eigenvalue:

$$
L_{g}(0) \mathbf{1}_{Z, g}=c_{Z, g} \mathbf{1}_{Z, g} .
$$

Also from Proposition 4.12,

$$
\left[L_{g}(0), a(m)\right]=-m a(m)
$$

for $a \in A$ and $m \in \frac{1}{2 N} \mathbb{Z}$. Thus the weight gradation of $C M(Z, g)$ coincides with the $L_{g}(0)$-eigenspace decomposition. Now from (4.15), (4.17), (4.42) and (4.43) we conclude:

Proposition 4.16. The graded-dimensions of $C M^{r}(Z, g)$ are given by:

(1) Take $0 \leqq n_{i, g}<N$ for $i=1, \ldots$, l. If some $n_{i, g}=0$,

$$
\operatorname{ch}_{q} C M^{r}(\mathbb{Z}, g)=\frac{1}{2} q^{c_{\mathbb{Z}, g}-l / 24} \prod_{i=1}^{l} \prod_{n>0}\left(1+q^{-n_{l, g} / N+1+n}\right)\left(1+q^{n_{l, g} / N+n}\right) ;
$$

if all $n_{i, g} \neq 0$,

$$
\begin{aligned}
\operatorname{ch}_{q} C M^{r}(\mathbb{Z}, g)= & \frac{1}{2} q^{c_{\mathbb{Z}, g}-l / 24}\left(\prod_{i=1}^{l} \prod_{n>0}\left(1+q^{-n_{i, g} / N+1+n}\right)\left(1+q^{n_{i, g} / N+n}\right)\right. \\
& +(-1)^{r}\left(\prod_{i=1}^{l} \prod_{n>0}\left(1-q^{-n_{t, g} / N+1+n}\right)\left(1-q^{n_{t, g} / N+n}\right)\right) .
\end{aligned}
$$

(2) Take $-N / 2 \leqq n_{i, g} \leqq N / 2$. If some $n_{i, g}=N / 2$,

$$
\operatorname{ch}_{q} C M^{r}\left(\mathbb{Z}+\frac{1}{2}, g\right)=\frac{1}{2} q^{c_{\mathbb{Z}+1 / 2, g}-l / 24} \prod_{i=1}^{l} \prod_{n>0}\left(1+q^{-n_{l, g} / N+1 / 2+n}\right)\left(1+q^{n_{l, g} / N+1 / 2+n}\right) ;
$$

if all $n_{i, g} \neq N / 2$,

$$
\begin{aligned}
\operatorname{ch}_{q} C M^{r}\left(\mathbb{Z}+\frac{1}{2}, g\right)= & \frac{1}{2} q^{c_{\mathbb{Z}, g}-l / 24}\left(\prod_{i=1}^{l} \prod_{n>0}\left(1+q^{-n_{l, g} / N+1 / 2+n}\right)\left(1+q^{n_{t, g} / N+1 / 2+n}\right)\right. \\
& +(-1)^{r} \prod_{i=1}^{l} \prod_{n>0}\left(1-q^{-n_{l, g} / N+1 / 2+n}\right)\left(1+q^{n_{i, g} / N+1 / 2+n}\right) .
\end{aligned}
$$

\section{Boson-Fermion Correspondences}

We have constructed irreducible $\hat{\mathfrak{g}}[g]$-modules $C M^{r}(Z, g)$ for $g \in G$ in the last section. In this section we shall realize these modules as $V_{L_{g}+\lambda_{1, g}+\beta_{g}}$ constructed in 
Sect. 3, where $\beta_{g} \in\left(\mathbf{h}_{g}\right)_{\mathbb{Q}}$ depends on $g$ and $\lambda_{i, g} \in L_{g}^{\circ}$ for $i=0,1, l-1, l$ (see (5.2) and (5.3) below). We then establish the isomorphisms between these modules - the boson-fermion correspondence. Realizing $G$ as a group of automorphisms of the VOA $V_{L}$, we show that $C M^{r}(Z, g)$ are irreducible $g$-twisted modules of the VOA $C M^{0}\left(\mathbb{Z}+\frac{1}{2}\right)$ via the boson-fermion correspondence.

Since $g \in G$ is an inner automorphism of $\mathfrak{g}$, the $g$-twisted affine algebra $\hat{\mathfrak{g}}[g]$ is isomorphic to $\hat{\mathfrak{g}}$ (see $[\mathrm{K}]$ ). Moreover, we see the four level 1 standard modules as $C M^{r}(Z, g)$ for $r=0,1$ and $Z=\mathbb{Z}, \mathbb{Z}+\frac{1}{2}$ in the fermionic picture by Theorem 4.13. On the other hand, there are also four level 1 standard $\hat{\mathfrak{g}}[g]$-modules from the bosonic picture in Sect. 3. For $g \in G$ we define a number $\varepsilon_{g}$ as follows

$$
\varepsilon_{g}= \begin{cases}0 \text { if } \operatorname{dim} \sum_{k \leqq N / 2} & A_{k, g}^{+} \text {is even } \\ 1 & \text { otherwise }\end{cases}
$$

Set

$$
\beta_{g}=-\frac{1}{N} \sum_{i=1}^{l} n_{i, g} h_{i, g}+\varepsilon_{g} h_{l, g} \in \mathfrak{h}_{g},
$$

where $n_{i, g}$ is as in (4.3) with $N>n_{1, g} \geqq \cdots \geqq n_{l, g} \geqq 0$. Then $g-\beta_{g}$ is an automorphism of $V_{L_{g}}$ by Proposition 3.3 (1). By identifying the weight 1 subspace $\left(V_{L_{g}}\right)_{1}$ of $V_{L_{g}}$ with $\mathrm{g}$, the restriction of $g_{-\beta_{g}}$ to $\left(V_{L_{g}}\right)_{1}$ is $g$ (see Remarks 3.2 and 3.6). According to Theorem 3.4 there are four irreducible $g_{-\beta_{g}}$-twisted $V_{L_{g}}$-modules $V_{L_{g}+\lambda_{i, g}+\beta_{g}}$ (for $i=0,1, l-1, l)$ which are also the level 1 standard $\hat{\mathrm{g}}[g]$-modules, where

$$
\begin{gathered}
\lambda_{0, g}=0, \quad \lambda_{1, g}=h_{1, g}, \\
\lambda_{l-1, g}=\frac{1}{2}\left(h_{1, g}+\cdots+h_{l-2, g}+h_{l-1, g}-h_{l, g}\right), \\
\lambda_{l, g}=\frac{1}{2}\left(h_{1, g}+\cdots+h_{l-2, g}+h_{l-1, g}+h_{l, g}\right) .
\end{gathered}
$$

Recall that, as vector spaces, $V_{L_{g}+\lambda_{1, g}+\beta_{g}}=V_{L_{g}}$. Set

$$
b_{i, g}=l\left(c_{i, g}\right) \in V_{L_{g}+\lambda_{i, g}+\beta_{g}}
$$

for $c_{i, g} \in \hat{L}_{g}$ for $i=0,1, l-1, l$ so that

$$
\bar{c}_{0 . g}=\sum_{n_{i, g} \geqq N / 2} h_{i, g}-\varepsilon_{g} h_{l, g}, \quad \bar{c}_{1, g}=\bar{c}_{0, g}, \quad \bar{c}_{l-1, g}=0, \quad \bar{c}_{l, g}=-2 \varepsilon_{g} h_{g, l} .
$$

Also set $f_{0, g}=\mathbf{1}_{\mathbb{Z}+\frac{1}{2}, g}, f_{1, g}=a_{1, g}(-1 / 2)$ if $n_{1}=0, f_{1, g}=a_{1, g}\left(n_{1} / N-3 / 2\right)$ if $n_{1} \neq 0$, $f_{l-1, g}=a_{l, g}^{*}\left(-n_{l} / N\right)$ and $f_{l, g}=\mathbf{1}_{\mathbb{Z}, g}$. We have the following boson-fermion corrrespondence:

Theorem 5.1. There are unique isomorphisms of $\hat{\mathrm{g}}[\mathrm{g}]$-modules $\sigma_{g}$ :

$$
\begin{aligned}
V_{L+\beta_{g}} & \simeq C M^{0}\left(\mathbb{Z}+\frac{1}{2}, g\right), \quad V_{L+\lambda_{1, q}+\beta_{g}} \simeq C M^{1}\left(\mathbb{Z}+\frac{1}{2}, g\right), \\
V_{L+\lambda_{l, g}+\beta_{g}} & \simeq C M^{\varepsilon_{g}}(\mathbb{Z}, g), \quad V_{L+\lambda_{l-1, g}+\beta_{g}} \simeq C M^{1+\varepsilon_{g}}(\mathbb{Z}, g)
\end{aligned}
$$

such that

$$
\sigma_{g} x(z) \sigma_{g}^{-1}=x(z)
$$


for $x \in \hat{\mathfrak{g}}$,

$$
\sigma_{g} b_{i, g}=f_{i, g}
$$

for $i=0,1, l-1, l$ if $\varepsilon_{g}=0$ and

$$
\sigma_{g} b_{i, g}=f_{i, g}(i=0,1), \quad \sigma_{g} b_{l-1, g}=f_{l, g}, \quad \sigma_{g} b_{l, g}=f_{l-1, g},
$$

if $\varepsilon_{g}=1$, where $\varepsilon_{g}+r$ is taken modulo 2 .

Proof. We shall prove the theorem by giving explicit isomorphisms between $\hat{\mathrm{g}}$ and $\hat{\mathrm{g}}[g]$ so that $b_{i, g}$ and $f_{i, g}$ are highest weight vectors with the same highest weights for $i=0,1, l-1, l$. It is well-known that the level 1 standard $\hat{\mathfrak{g}}$-modules are parametrized by four dominant integral weights $\lambda=\lambda_{i, g} \in \mathfrak{h}_{g}^{*}$ for $i=0,1, l-1, l$ such that the highest weight of $\hat{\mathfrak{g}}$-module, viewed as a linear form on $\mathfrak{h}_{g} \oplus \mathbb{C} c \subset \hat{\mathfrak{g}}$, is given by $\lambda$, sending $c$ to 1 . We denote the corresponding level one standard $\hat{\mathfrak{g}}$-module by $L(1, \lambda)$.

The first isomorphism $\phi_{1}$ from $\hat{\mathfrak{g}}$ to $\hat{\mathfrak{g}}[g]$ is given by:

$$
\begin{aligned}
c & \mapsto c, \\
x_{i j, g}(m) & \mapsto x_{i j, g}\left(m+n_{i, g} / N+n_{j, g} / N\right), \\
x_{i j, g}^{*}(m) & \mapsto x_{i j, g}^{*}\left(m+n_{i, g} / N-n_{j, g} / N\right)+\delta_{i, j} \delta_{m, 0} n_{i, g} / N c, \\
x_{i j, g}^{* *}(m) & \mapsto x_{i j, g}^{* *}\left(m-n_{i, g} / N-n_{j, g} / N\right) .
\end{aligned}
$$

for $m \in \mathbb{Z}$. Recall from Remark 3.6 that the action of $\hat{\mathfrak{g}}[g]$ on $V_{L_{g}+\lambda_{t, g}+\beta_{g}}$ is given by $x(z)=Y_{\lambda_{1, g}+\beta_{g}}(x, z)$. In particular, the action of $\mathfrak{h}_{g}$ is given by (3.17). It is straightforward to check the following for $k=l-1, l$ :

$$
\begin{gathered}
\phi_{1}\left(x_{i j, g}(0)\right) b_{k, g}=\phi_{1}\left(x_{i j, g}^{*}(0)\right) b_{k, g}=\phi_{1}\left(x_{1 l, g}^{* *}(1)\right) b_{k, g}=0, \quad \text { for } i<j, \\
\phi_{1}(\alpha) b_{k, g}=\left\langle\lambda_{k, g}, \alpha\right\rangle b_{k, g} \quad \text { if } \varepsilon_{g}=0, \\
\phi_{1}(\alpha) b_{l-1, g}=\left\langle\lambda_{l, g}, \alpha\right\rangle b_{l-1, g}, \quad \phi_{1}(\alpha) b_{l, g}=\left\langle\lambda_{l-1, g}, \alpha\right\rangle b_{l, g} \quad \text { if } \varepsilon_{g}=1 .
\end{gathered}
$$

for $\alpha \in \mathfrak{h}_{g}$. The same relations hold for $f_{k, g}$ with $k=l-1, l$ by using (4.27). That is, $b_{k, g}$ and $f_{k, g}$ are highest weight vectors with the same highest weights for $k=l-1, l$. This proves the theroem in these cases. In particular,

$$
\begin{aligned}
V_{L_{g}+\lambda_{l-1}+\beta_{g}} & \simeq C M^{1+\varepsilon_{g}}(\mathbb{Z}, g) \simeq L\left(1,\left(1-\varepsilon_{g}\right) \lambda_{l-1, g}+\varepsilon_{g} \lambda_{l, g}\right), \\
V_{L_{g}+\lambda_{l}+\beta_{g}} & \simeq C M^{\varepsilon_{g}}(\mathbb{Z}, g) \simeq L\left(1, \varepsilon_{g} \lambda_{l-1, g}+\left(1-\varepsilon_{g}\right) \lambda_{l, g}\right) .
\end{aligned}
$$

Similarly for the remaining cases. But this time use the isomorphim $\phi_{2}$ from $\hat{\mathfrak{g}}$ to $\hat{\mathrm{g}}[g]$ given by

$$
\begin{aligned}
c & \mapsto c, \\
x_{i j, g}(m) & \mapsto x_{i j, g}\left(m+n_{i}^{\prime} / N+n_{j, g}^{\prime} / N\right), \\
x_{i j, g}^{*}(m) & \mapsto x_{i j, g}^{*}\left(m+n_{i}^{\prime} / N-n_{j, g}^{\prime} / N\right)+\delta_{i, j} \delta_{m, 0} n_{i, g}^{\prime} / N c, \\
x_{i j, g}^{* *}(m) & \mapsto x_{i j, g}^{* *}\left(m-n_{i, g}^{\prime} / N-n_{j, g}^{\prime} / N\right) .
\end{aligned}
$$

for $m \in \mathbb{Z}$ where $n_{i, g}^{\prime}=n_{i, g}$ if $n_{i, g}<N / 2$ and $n_{i, g}^{\prime}=n_{i, g}-N$ if $n_{i, g} \geqq N / 2$. In these cases the results are:

$$
V_{L_{g}+\lambda_{k}+\beta_{g}} \simeq C M^{k}(\mathbb{Z}, g) \simeq L\left(1, \lambda_{k}\right)
$$

for $k=0,1$. 
Corollary 5.2. We have the following q-identities:

$$
\frac{\theta_{L_{i_{g}+\lambda_{i, g}+\beta_{g}}(q)}}{\eta(q)^{l}}=\operatorname{ch}_{q} C M^{i}(\mathbb{Z}, g)
$$

for $i=0,1$ and

$$
\begin{gathered}
\frac{\theta_{L_{\lambda_{g}+\lambda_{l-1 g}+\beta_{g}}(q)}}{\eta(q)^{l}}=\operatorname{ch}_{q} C M^{1+\varepsilon_{g}}\left(\mathbb{Z}+\frac{1}{2}, g\right), \\
\frac{\theta_{L_{\lambda_{g}+\lambda_{l, g}+\beta_{g}}(q)}}{\eta(q)^{l}}=\operatorname{ch}_{q} C M^{\varepsilon_{g}}\left(\mathbb{Z}+\frac{1}{2}, g\right) .
\end{gathered}
$$

Proof. From Remarks 3.2 and 3.6, formula (3.27), Theorem 5.1, it is enough to prove that wt $b_{i, g}=\mathrm{wt} \sigma_{g}\left(b_{i, g}\right)$ for $i=0,1, l-1, l$. Now a straightforward calculation of weights of these vectors gives the result.

Recall that $L=L_{1}$ is the root lattice of $\mathrm{g}$ corresponding to the identity automorphism. Our next goal is to realize $G$ as a group of automorphisms of $V_{L}$ so that $V_{L_{g}+\lambda_{l, g}+\beta}$ will be an irreducible $g$-twisted $V_{L}$-module. The main idea is to identify $V_{L}$ with $V_{L_{g}}$ as vertex operator algebras. Let $\tau_{g}: V_{L_{g}} \rightarrow C M^{0}\left(\mathbb{Z}+\frac{1}{2}\right)$ be the unique $\hat{\mathfrak{g}}$-module isomorphism such that

$$
\tau_{g} \mathbf{1}=\mathbf{1}_{\mathbb{Z}+\frac{1}{2}}, \quad \tau_{g} x(z) \tau_{g}^{-1}=x(z)
$$

on $C M^{0}\left(\mathbb{Z}+\frac{1}{2}\right)$ for $x \in \mathfrak{g}$. We also assume that $b_{0,1}=\mathbf{1}$. Then $\tau_{1}=\sigma_{1}$. More precisely we have:

$$
\tau_{g}\left(x_{1}\left(m_{1}\right) \ldots x_{k}\left(m_{k}\right) \mathbf{1}\right)=x_{1}\left(m_{1}\right) \ldots x_{k}\left(m_{k}\right) \mathbf{1}_{\mathbb{Z}+\frac{1}{2}}
$$

for $x_{i} \in \mathfrak{g}$ and $m_{i} \in \mathbb{Z}$. Since both $V_{L_{g}}$ and $C M^{0}\left(\mathbb{Z}+\frac{1}{2}\right)$ are VOAs (see Theorem 3.1 and Remark 4.14) it is natural to expect that $\tau_{g}$ is an isomorphism of VOAs. Note that the Virasoro element of $V_{L_{g}}$ is given by

$$
\omega_{g}=\frac{1}{2} \sum_{i=1}^{l} h_{i, g}(-1)^{2}
$$

and the Virasoro element of $C M^{0}\left(\mathbb{Z}+\frac{1}{2}\right)$ is $\omega$ given in Remark 4.14. We still denote by $\omega_{g}$ the image of $\tau_{g}\left(\omega_{g}\right)$ in $C M^{0}\left(\mathbb{Z}+\frac{1}{2}\right)$, that is

$$
\omega_{g}=\frac{1}{2} \sum_{k=1}^{l} h_{k, g}(-1)^{2} \mathbf{1}_{\frac{1}{2}+\mathbb{Z}} \in C M^{0}\left(\frac{1}{2}+\mathbb{Z}\right) .
$$

Lemma 5.3. The element $\omega_{g}$ is independent of $g$, that is, $\omega_{g}=\omega$.

Proof. Note that

$$
\omega_{g}=-\frac{1}{2} \sum_{k=1}^{l}\left(a_{k, g}^{*}(-3 / 2) a_{k, g}(-1 / 2)+a_{k, g}(-3 / 2) a_{k, g}^{*}(-1 / 2)\right) \mathbf{1}_{1 / 2+\mathbb{Z}} .
$$

Now the result follows.

Remark 5.4. Since both $V_{L_{g}}$ and $C M^{0}\left(\mathbb{Z}+\frac{1}{2}\right)$ are generated by $\mathfrak{g}$ which is the weight one subspace in the sense of [FLM2], we see that $\tau_{g}$ is an isomorphism of VOAs. Clearly, the VOA structure of $C M^{0}\left(\mathbb{Z}+\frac{1}{2}\right)$ is independent of $g \in G$. We identify $V_{L}$ with $V_{L_{g}}$ via $\tau_{g}^{-1} \tau_{1}$. 
Recall from Remark 4.15 that $G$ acts on $C M^{0}\left(\mathbb{Z}+\frac{1}{2}\right)$ as a group of VOA automorphism. Thus $G$ acts on $V_{L}$ by

$$
g\left(x_{1}\left(m_{1}\right) \ldots x_{k}\left(m_{k}\right) \mathbf{1}\right)=\left(g x_{1}\right)\left(m_{1}\right) \ldots\left(g x_{k}\right)\left(m_{k}\right) \mathbf{1}
$$

(for $x_{i} \in \mathfrak{g}$ and $m_{i} \in \mathbb{Z}$ ) as a group of automorphisms of $V_{L}$. That is, $g Y(v, z) g^{-1}=$ $Y(g v, z)$ for $g \in G$ and $u \in V_{L}$. Moreover, using the identification of $V_{L}$ with $V_{L_{g}}$ and Theorem 3.4 we have:

Proposition 5.5. The spaces $V_{L_{g}+\lambda_{l, g}+\beta_{g}}($ for $i=0,1, l-1, l)$ are irreducible $g$-twisted $V_{L}$-modules and are unique up to isomorphism.

Now we return to the fermionic picture. Set

$$
M_{g}=C M\left(\mathbb{Z}+\frac{1}{2}, g\right) \oplus C M(\mathbb{Z}, g) .
$$

We can define a linear map

$$
\begin{aligned}
C M\left(\mathbb{Z}+\frac{1}{2}\right) & \rightarrow\left(\text { End } M_{g}\right)\left[\left[z^{1 / N}, z^{-1 / N}\right]\right] \\
v & \mapsto Y_{g}(u, z)=\sigma_{g} Y_{g}\left(\sigma_{1}^{-1}(v), z\right) \sigma_{g}^{-1}=\sigma_{g} Y_{g}\left(\tau_{g}^{-1}(v), z\right) \sigma_{g}^{-1},
\end{aligned}
$$

where we use $Y_{g}(u, z)$ for $u \in V_{L_{g}}$ to denote $Y_{\lambda_{l, g}+\beta_{g}}(u, z)$ on $V_{L_{g}+\lambda_{t, g}+\beta_{g}}$ for $i=0,1$, $l-1, l$ (see (3.18)). By Remark 3.6, Theorem 5.1 and Proposition 5.5 we have

Proposition 5.6. The space $\left(M_{g}, Y_{g}\right)$ is a g-twisted $C M^{0}\left(\mathbb{Z}+\frac{1}{2}\right)$-module and the four spaces $C M^{r}(Z, g)$ are the unique irreducible $g$-twisted $C M^{0}\left(\mathbb{Z}+\frac{1}{2}\right)$-modules.

Recall from 4.3 that $C_{G}(g)$ acts on $A(Z, g), C M(Z, g)$ such that $h \mathbf{1}_{Z, g}=\mathbf{1}_{Z, g}$ and that $h a(z) h^{-1}=(h a)(z)$ for $h \in C_{G}(g)$ and $a \in A$. Also recall (2.20).

Theorem 5.7. Let $g, h \in G$. Then there exists an invertible linear map $\phi(h): M_{h^{-1} g h} \rightarrow M_{g}$ such that $\phi(h)\left(C M^{r}\left(Z, h^{-1} g h\right)\right)=C M^{r}(Z, g)$ and that

$$
\phi(h) Y_{h^{-1} g h}(u, z) \phi(h)^{-1}=Y_{g}(h u, z)
$$

for $u \in C M^{0}\left(\mathbb{Z}+\frac{1}{2}\right)$. In particular, $\phi(h)$ gives an isomorphism of $h^{-1}$ gh-twisted $C M^{0}\left(\mathbb{Z}+\frac{1}{2}\right)$-modules $C M^{r}\left(Z, h^{-1} g h\right)$ and $h \circ C M^{r}(Z, g)$. In the case $h \in C_{G}(h)$, we may take $\phi(h)=h$ and (5.17) becomes

$$
h Y_{g}(u, z) h^{-1}=Y_{g}(h u, z) .
$$

Proof. First we define the map $\phi(h)$. Recall $A(Z, g)$ and $A^{ \pm}(Z, g)$ from (4.9) and (4.13). Define an invertible map $\phi(h)$ from $A\left(Z, h^{-1} g h\right)$ to $A(Z, g)$ by:

$$
\phi(h)(a(n))=(h a)(n)
$$

for $a(n) \in A\left(Z, h^{-1} g h\right)$, where $a \in A$ and $n \in \mathbb{Q}$. Then $\phi(h)$ preserves the polarization $\phi(h) A^{ \pm}\left(Z, h^{-1} g h\right)=A^{ \pm}(Z, g)$. If we extend $\phi(h)$ to an algebra isomorphism from $C\left(A\left(Z, h^{-1} g h\right)\right)$ to $c(A(Z, g))$ then this induces an invertible linear map

$$
\phi(h): C M\left(Z, h^{-1} g h\right) \rightarrow C M(Z, g)
$$

such that $\phi(h) C M^{r}\left(Z, h^{-1} g h\right)=C M^{r}(Z, g)$. In fact, $\phi(h)$ has the following explicit action:

$$
\phi(h)\left(a_{1}\left(n_{1}\right) \ldots a_{k}\left(n_{k}\right) \mathbf{1}_{Z, h^{-1} g h}\right)=\left(h a_{1}\right)\left(n_{1}\right) \ldots\left(h a_{k}\right)\left(n_{k}\right) \mathbf{1}_{Z, g}
$$


for $a_{i} \in A$ and $n_{i} \in \mathbb{Q}$. Now it is clear from (4.27) and Theorem 4.9 that (5.17) holds for $u \in \mathfrak{g}$ (which is identified with the weight one subspace of $C M\left(\mathbb{Z}+\frac{1}{2}\right)$ ).

Next we prove (5.17) for arbitrary $u \in C M^{0}\left(\mathbb{Z}+\frac{1}{2}\right)$. The idea of the proof is to prove that $Y_{g}(u, z)$ can be expressed in terms of $Y_{g}(v, z)$ for $v \in \mathfrak{g}$. For $u=$ $x(-n)\left(=x(-n) \mathbf{1}_{\mathbb{Z}+\frac{1}{2}}\right) \in C M^{0}\left(\mathbb{Z}+\frac{1}{2}\right) \quad$ with $n \in \mathbb{Z} \quad$ positive we rewrite $u=\frac{1}{(n-1) !} L(-1)^{n-1} x(-1)$. Then

$$
Y(x(-n), z)=\frac{1}{(n-1) !}\left(\frac{d}{d z}\right)^{n-1} Y(x(-1), z) .
$$

Now let $u=x(-n) \cdot \omega \in C M^{0}\left(\mathbb{Z}+\frac{1}{2}\right)$ for some $w$. We also assume that $x \in \mathfrak{g}_{i}$. Then from the $g$-twisted Jacobi identity (2.18) we have

$$
\begin{gathered}
z_{0}^{-1} \delta\left(\frac{z_{1}-z_{2}}{z_{0}}\right) Y_{g}\left(x(-1), z_{1}\right) Y_{g}\left(w, z_{2}\right)-z_{0}^{-1} \delta\left(\frac{z_{2}-z_{1}}{-z_{0}}\right) Y_{g}\left(w, z_{2}\right) Y_{g}\left(x(-1), z_{1}\right) \\
=z_{2}^{-1}\left(\frac{z_{1}-z_{0}}{z_{2}}\right)^{-i / N} \delta\left(\frac{z_{1}-z_{0}}{z_{2}}\right) Y_{g}\left(Y\left(x(-1), z_{0}\right) w, z_{2}\right) .
\end{gathered}
$$

Multiplying (5.21) by $z_{0}^{-n}$ and taking $\operatorname{Res}_{z_{0}}$ we get

$$
\begin{gathered}
z_{2}^{-1} \sum_{k \geqq 0} \frac{\left(-\frac{\partial}{\partial z_{1}}\right)^{k}}{k !}\left(\left(\frac{z_{1}}{z_{2}}\right)^{-i / N} \delta\left(\frac{z_{1}}{z_{2}}\right)\right) Y_{g}\left(x(-n+k) w, z_{2}\right) \\
=\left(z_{1}-z_{2}\right)^{-n} Y_{g}\left(x(-1), z_{1}\right) Y_{g}\left(w, z_{2}\right)-(-1)^{n}\left(z_{2}-z_{1}\right)^{-n} Y\left(w, z_{2}\right) Y_{g}\left(x(-1), z_{1}\right) .
\end{gathered}
$$

Now we multiply (5.22) by $z_{1}^{i / N} z_{2}^{-i / N}$ and take $\operatorname{Res}_{z_{1}}$ to obtain

$$
\begin{gathered}
Y_{g}\left(x(-n) w, z_{2}\right) \\
=-\operatorname{Res}_{z_{1}}\left\{z_{2}^{-1}\left(\frac{z_{1}}{z_{2}}\right)^{i / N} \sum_{k \geqq 0} \frac{\left(-\frac{\partial}{\partial z_{1}}\right)^{k}}{k !}\left(\left(\frac{z_{1}}{z_{2}}\right)^{-i / N} \delta\left(\frac{z_{1}}{z_{2}}\right)\right) Y_{g}\left(x(-n+k) w, z_{2}\right)\right. \\
+\left(\frac{z_{1}}{z_{2}}\right)^{i / N}\left(\left(z_{1}-z_{2}\right)^{-n} Y_{g}\left(x(-1), z_{1}\right) Y_{g}\left(w, z_{2}\right)\right. \\
\left.-(-1)^{n}\left(z_{2}-z_{1}\right)^{-n} Y_{g}\left(w, z_{2}\right) Y_{g}(x(-1), z)\right\} .
\end{gathered}
$$

Thus $y_{g}(h(x(-n) w), z)$ can be expressed by the operators $Y_{g}(h x(-1), z), Y_{g}(h w, z)$ and $Y_{g}(h(x(-n+k) w), z)$ for $k>0$. Similarly, $Y_{h^{-1}} g h(x(-n) w, z)$ can be expressed by the operators $Y_{h^{-1} g h}(x(-1), z), Y_{h^{-1} g h}(w, z)$ and $Y_{h^{-1} g h}(x(-n+k) w, z)$ for $k>0$ with the same relation. This shows that if $(5.17)$ holds for $w$ and $x(-n+k) w(k>0)$ then (5.17) also holds for $x(-n) w$.

Let $u=x_{1}\left(-n_{1}\right) \ldots x_{l}\left(-n_{s}\right) \in C M^{0}\left(\mathbb{Z}+\frac{1}{2}\right)$ for $g$-homogeneous $x_{i} \in \mathfrak{g}$ and $n_{i}>0$. Now it is straightforward to show by induction on $s$ and $n_{1}$ that (5.17) holds for $u$. The other assertions are clear.

Corollary 5.8. $C M^{0}\left(\mathbb{Z}+\frac{1}{2}\right)$ is a rational VOA, $G$ is a group of inner automorphisms of $C M^{0}\left(\mathbb{Z}+\frac{1}{2}\right)$, and properties SR1, SR2 of Sect. 2 are satisfied (where all projective representations are ordinary linear representations). 
Using the isomorphism $\sigma_{g}$ of Theorem 5.1 and the invertible map $\phi(h)$ of Theorem 5.1 we define an invertible linear map $\phi(h)$ from $V_{L_{h^{-1}} g h}+\lambda_{i, h^{-1} g h}+\beta_{h^{-1} g h}$ to $V_{L_{g}+\lambda_{r, g}+\beta_{g}}$ for $i=0,1, l-1, l$ by

$$
\phi(h) w=\sigma_{g}^{-1} \phi(h) \sigma_{h^{-1}} g h(w)
$$

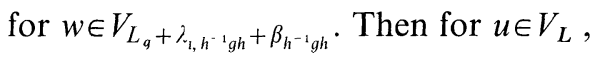

$$
\begin{aligned}
\phi(h) Y_{h^{-1} g h}(u, z) \phi(h)^{-1}(w) & =\phi(h) Y_{h^{-1} g h}(u, z) \sigma_{h^{-1} g h}^{-1} \phi(h)^{-1} \sigma_{g}(w) \\
& =\phi(h) \sigma_{h^{-1} g h}^{-1} Y_{h^{-1} g h}\left(\sigma_{1} u, z\right) \phi(h)^{-1} \sigma_{g}(w) \\
& =\sigma_{g}^{-1} \phi(h) Y_{h^{-1} g h}\left(\sigma_{1} u, z\right) \phi(h)^{-1} \sigma_{g}(w) \\
& =\sigma_{g}^{-1} Y_{g}\left(h \sigma_{1} u, z\right) \sigma_{g}(w) \\
& =\sigma_{g}^{-1} Y_{g}\left(\sigma_{1} h u, z\right) \sigma_{g}(w) \\
& =Y_{g}(h u, z)(w) .
\end{aligned}
$$

This concludes the proof of

Proposition 5.9. Let $g, h \in G$. Then there exists an invertible linear map $\phi(h)$ :

$$
V_{L_{h^{-1} g h}+\lambda_{1, h^{-1} g h}+\beta_{h^{-1} g h}} \rightarrow V_{L_{g}+\lambda_{1, g}+\beta_{g}}
$$

for $i=0,1, l-1, l$ such that

$$
\phi(h) Y_{h^{-1} g h}(u, z) \phi(h)^{-1}=Y_{g}(h u, z)
$$

for $u \in V_{L}$. In particular, $\phi(h)$ gives an isomorphism of $h^{-1}$ gh-twisted $V_{L}$-modules from $V_{L_{h^{-1} g}+\lambda_{1, h^{-1} g h}+\beta_{h^{-1} g h}}$ to $h \circ V_{L_{g}+\lambda_{1, g}+\beta_{g}}$. In case $h \in C_{G}(h)$, we may take $\phi(h)=h$ and (5.24) becomes

$$
h Y_{g}(u, z) h^{-1}=Y_{g}(h u, z) .
$$

Moreover, there are commuting diagrams:

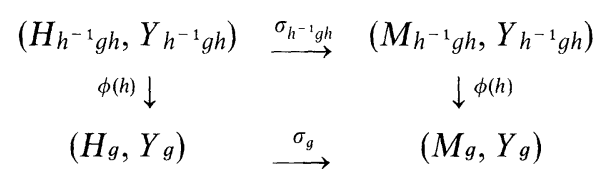

where

$$
H_{g}=\bigoplus_{i=0,1, l-1, l} V_{L_{g}+\lambda_{i, g}+\beta_{g}}
$$

\section{Twisted Modules for $V_{\Gamma_{l}}$}

In this section we shall continue our discussion in Sects. 4 and 5 with $l$ divisible by 8. We realize $G$ as a group of automorphisms of the holomorphic vertex operator algebra $V_{\Gamma_{l}}$ such that $V_{\Gamma_{l, g}+\beta_{g}}$ is the unique irreducible $g$-twisted module. Using the boson-fermion correspondence we get a holomorphic vertex operator algebra $C M^{0}\left(\mathbb{Z}+\frac{1}{2}\right) \oplus C M^{0}(\mathbb{Z})$ and its unique irreducible $g$-twisted module $C M^{0}\left(\mathbb{Z}+\frac{1}{2}, g\right) \oplus C M^{\varepsilon}(\mathbb{Z}, g)$. 
In this section we assume that $G$ is a subgroup of $S L(l, \mathbb{R})$. The following lemma then is obvious.

Lemma 6.1. If $\eta^{n_{1}}, \ldots, \eta^{n_{l}}$ are the eigenvalues of $g \in G$ on $A^{+}$, then $n_{1}+\ldots+$ $n_{l} \equiv 0 \bmod N$.

Recall from (4.7) that $\Gamma_{l, g}=L_{g} \cup\left(L_{g}+\lambda_{l, g}\right)$. Then $V_{\Gamma_{l, g}}=V_{L_{g}} \oplus V_{L_{g}+\lambda_{l, g}}$. From Proposition 4.1 (3) we know that $\lambda_{l, g}=\lambda_{l}$, that is, $\lambda_{l} \in \bigcap_{g \in G} \Gamma_{l, g}$. Let $a_{g} \in \Gamma_{l, g}$ such that $\bar{a}_{g}=\lambda_{l}$. Since $l\left(a_{g}^{2}\right) \in V_{L_{g}}$ which is identified with $V_{L}$, we can assume that $\imath\left(a_{g}^{2}\right)=l\left(a^{2}\right)$ (where again we write $\left.a=a_{1}\right)$. Then $l\left(a_{g}\right)$ is a highest weight vector of $V_{L_{g}}+\lambda_{l}$. For $g \in G$ set

$$
V(g)=C M^{0}\left(\mathbb{Z}+\frac{1}{2}, g\right) \oplus C M^{\varepsilon_{g}}(\mathbb{Z}, g) \otimes N_{g},
$$

where $\varepsilon_{g}$ is defined in (5.1) and $N_{g}=\mathbb{C}$ is a one-dimensional $C_{G}(g)$-module affording the linear character

$$
\chi_{g}(h)=\operatorname{det}\left(\left.h\right|_{\left.\oplus_{k \leqq N / 2} A_{k}^{+}\right)^{-1}}=e^{2 \pi i\left\langle\beta_{h,} \bar{c}_{0, g}+\varepsilon_{g} h_{l, g}\right\rangle}\right.
$$

for $h \in C_{G}(g)$ (see (5.5) for the notation $\bar{c} 0, g$ ). In other words we modify the action of $C_{G}(g)$ on $C M^{\varepsilon_{g}}(\mathbb{Z}, g)$ by $\chi_{g}$. In particular $V(1)=C M^{0}\left(\mathbb{Z}+\frac{1}{2}\right) \oplus C M^{0}(\mathbb{Z})$. From Propositon 5.1, we have a unique $\hat{\mathrm{g}}$-module isomorphism $\tau_{g}: V_{\Gamma_{l, g}} \simeq V(1)$ such that $\tau_{g}(\mathbf{1})=\mathbf{1}_{\mathbb{Z}+\frac{1}{2}, g}, \tau_{g}\left(l\left(a_{g}\right)\right)=\mathbf{1}_{\mathbb{Z}, g}$ and $\tau_{g} Y(u, z) \tau_{g}^{-1}=Y\left(\sigma_{1} u, z\right)$ for $u \in V_{L}$.

Proposition 6.2. There is an identification of VOAs $\tau_{g}^{-1} \tau_{1}: V_{\Gamma_{l}} \simeq V_{\Gamma_{l, g}}$ such that $V_{L}=V_{L_{g}}$ (as before) and $V_{L+\lambda_{l}}=V_{L_{g}+\lambda_{l, g}}$ with $\imath(a)=\imath\left(a_{g}\right)$.

Proof. As in Remark 5.4, $\tau=\tau_{1} \tau_{g}^{-1}$ is a $V_{L}$-module isomorphism from $V_{\Gamma_{l}}$ to $V_{\Gamma_{l, g}}$. In particular, we have the identification of VOAs $V_{L}$ and $V_{L_{g}}$. It is enough to prove that

$$
\tau Y(u, z) \tau^{-1}=Y(\tau u, z)
$$

for $u \in V_{L+\lambda_{l}}$. Let $U$ be a vertex operator algebra. The following skew-symmetry is well known (see, for example, [FHL]):

$$
Y\left(u_{1}, z\right) u_{2}=e^{L(-1) z} Y\left(u_{2},-z\right) u_{1}
$$

for $u_{i} \in U$. Let $v \in V_{L_{g}}=V_{L}$. Since $\left.\tau\right|_{V_{L}}=i d$, we see that

$$
\tau Y(u, z) \tau^{-1} v=\tau Y(u, z) v=\tau e^{L(-1) z} Y(v,-z) u=e^{L(-1) z} Y(v,-z) \tau(u) .
$$

On the other hand,

$$
Y(\tau(u), z) v=e^{L(-1) z} Y(v,-z) \tau(u) .
$$

That is, $\tau Y(u, z) \tau^{-1} v=Y(\tau(u), z) v$. Since $v \in V_{L}$ is arbitrary, (6.3) holds on $V_{L}$ for $u \in V_{L+\lambda_{l}}$.

Next let $v \in V_{L_{g}+\lambda_{l}}$. Then $Y(u, z) \tau^{-1}(v) \in V_{L}\left[\left[z, z^{-1}\right]\right]$ which is $\tau$-invariant. So we must prove the following relation:

$$
Y(u, z) \tau^{-1}(v)=Y(\tau(u), z) v .
$$

Note that

$$
Y\left(l\left(a_{g}\right), z\right)=E^{-}\left(-\lambda_{l}, z\right) E^{+}\left(-\lambda_{l}, z\right) a_{g} z^{\hat{\lambda}_{l}},
$$


where

$$
E^{ \pm}(\beta, z)=\exp \left(\sum_{n>0} \beta( \pm n) z^{\mp} /( \pm n)\right)
$$

for $\beta \in \mathfrak{h}_{g}$ (cf. (3.7)). Then

$$
Y(l(a), z) \tau^{-1} l\left(a_{g}\right)=E^{-}\left(-\lambda_{l}, z\right) z^{\left\langle\lambda_{l}, \lambda_{l}\right\rangle} l\left(a^{2}\right)=Y\left(l\left(a_{g}\right), z\right) l\left(a_{g}\right)
$$

and (6.5) holds for $u=l(a)$ and $v=l\left(a_{g}\right)$. Let $x \in \mathfrak{g}$. Then

$$
Y\left(x(-1), z_{1}\right) Y\left(l(a), z_{2}\right) l(a)=Y\left(x(-1), z_{1}\right) Y\left(l\left(a_{g}\right), z_{2}\right) l\left(a_{g}\right) .
$$

Using commutativity (2) of Proposition 2.3, we find that

$$
Y\left(l(a), z_{2}\right) Y\left(x(-1), z_{1}\right) l(a)=Y\left(l\left(a_{g}\right), z_{2}\right) Y\left(x(-1), z_{1}\right) l\left(a_{g}\right),
$$

or equivalently,

$$
Y\left(l(a), z_{2}\right) Y\left(x(-1), z_{1}\right) \tau^{-1}(l(a))=Y\left(\tau\left(l\left(a_{g}\right), z_{2}\right) Y\left(x(-1), z_{1}\right) l\left(a_{g}\right) .\right.
$$

Now by induction on $k$ we see that (6.5) holds for $u=\imath(a)$ and $v=x_{1}\left(n_{1}\right)$ $\ldots x_{k}\left(n_{k}\right) l\left(a_{g}\right)$ for $x_{i} \in \mathfrak{g}$ and $n_{i} \in \mathbb{Z}$. That is, (6.3) holds for $u=l(a)$. An argument similar to that in the proof of Theorem 5.7 show that (6.3) holds for all $u \in$ $V_{L+\lambda_{l}}$.

By Proposition 5.9, $G$ acts on $V_{\Gamma_{l}}$ in such a way that $g(\mathbf{1})=\mathbf{1}, g_{l}(a)=l(a)$ and $g Y(u, z) g^{-1}=Y(g(u), z)$ for $g \in G$ and $u \in V_{L}$. By the identification $V_{\Gamma_{l}}=V_{\Gamma_{l, g}}$ given in Proposition 6.2, we see that $g=g_{\beta_{q}}$. By Proposition 3.3 (1), we have

Proposition 6.3. $G$ is a group of automorphisms of $V_{\Gamma_{l}}$.

Since $\Gamma_{l, g}$ is a self-dual lattice (that is, $\Gamma_{l, g}^{\circ}=\Gamma_{l, g}$ ), by Theorem $3.4(2), V_{\Gamma_{l, q}+\beta_{g}}$ is the unique irreducible $g$-twisted $V_{\Gamma_{l, g}}$-module. Again by Proposition 6.2 we have:

Theorem 6.4. The space $V_{\Gamma_{l, g}+\lambda_{q}}$ is the unique irreducible g-twisted $V_{\Gamma_{l}}$-module.

Next we shall establish a result analogous to Theorem 5.7 for the VOA $V_{\Gamma_{1}}$. In order to do this we have to study the action of $G$ on the element $\imath\left(c_{0, g}\right) \in V_{L_{q}}$ (see (5.5)) in more detail.

Lemma 6.5. Let $g, h \in G$ such that $h\left(a_{l, h^{-1} g h}^{*}\right)=a_{l, g}^{*}$ if $\varepsilon_{g}=1$. Then

$$
h l\left(c_{0, h^{-1} g h}\right)=\lambda(g, h) l\left(c_{0, g}\right),
$$

where $\lambda(\cdot, \cdot) \in H^{1}\left(G, \mathbb{C}^{*}\right)$ is a 1-cocycle such that $\lambda(g, h)=\chi_{g}(h)^{-1}$ if $h \in C_{G}(g)$.

Proof. First let $\varepsilon_{g}=0$. Then $\varepsilon_{h^{-1}} g h=0$. Let $1 \leqq s \leqq l$ (necessarily even) such that $n_{i, g}$ for $1 \leqq i \leqq s$ is a complete list of $n_{i, g} \geqq N / 2$. Since $\operatorname{dim} \sum_{k \geqq N / 2} A_{k, g}^{+}=\operatorname{dim} \sum_{k \geqq N / 2}$

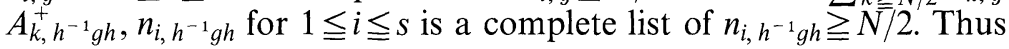

$$
l\left(c_{0, g}\right)=\mu_{g} x_{12, g}(-1) \ldots x_{s-1 s, g}(-1) l(1),
$$

where $\mu_{g} \in \mathbb{C}^{*}$ and $x_{i j, g}$ is defined in (4.34). Applying $\sigma_{1}$ given in Theorem 5.1 to $l\left(c_{0, g}\right)$, we find that

$$
\sigma_{1}\left(l\left(c_{0, g}\right)\right)=\mu_{g} a_{1, g}(-1 / 2) \ldots a_{s, g}(-1 / 2) \mathbf{1}_{\mathbb{Z}+\frac{1}{2}} \in C M\left(\mathbb{Z}+\frac{1}{2}\right) .
$$

We have the same expression for $\sigma_{1}\left(l\left(c_{0, h^{-1} g h}\right)\right)$ with $\mu_{g}$ and $a_{i, g}$ replaced by $\mu_{h^{-1} g h}$ and $a_{i, h^{-1} g h}$ respectively. Note that $h A_{k, h^{-1} g h}^{+}=A_{k, g}^{+}$for $k=0, \ldots, N-1$, and 
that $\Lambda^{\operatorname{dim}} \sum_{k \geqq N / 2} A_{k, g}\left(\sum_{k \geqq N / 2} A_{k, g}\right)$ is one-dimensional. We define $\lambda(h, g) \in \mathbb{C}^{*}$ by the following formula:

$$
h \sigma_{1}\left(l\left(c_{0, h^{-1} g h}\right)\right)=\lambda(g, h) \sigma_{1}\left(l\left(c_{0, g}\right)\right) .
$$

It is straightforward to verify that $\lambda(g, h)$ has the desired properties. Similarly for $\varepsilon_{g}=1$.

Let $g, h \in G$. From Proposition 5.9 we have an invertible map $\phi(h)$ from $V_{\Gamma_{l, h^{-1} g h}+\beta_{h^{-1} g h}}$ to $V_{\Gamma_{l, g}+\beta_{g}}$ such that $\phi(h) b_{0, h^{-1} g h}=b_{0, g}, \phi(h) b_{l, h^{-1} g h}=\sigma_{g}^{-1} h \sigma_{g} b_{l, g}$ (cf. (5.20)) and that $\phi(h) Y_{h^{-1} g h}(u, z) \phi(h)^{-1}=Y_{g}(h u, z)$ for $u \in V_{L}$. It is natural to expect that the last inequality holds also for all $v \in V_{\Gamma_{l}}$. For this purpose we now modify $\phi(h)$ in the following way:

We have:

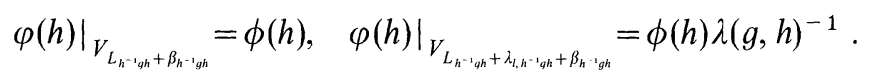

Theorem 6.6. Let $g, h \in G$. Suppose that $h\left(a_{l, h^{-1} g h}^{*}\right)=a_{l, g}^{*}$ if $\varepsilon_{g}=1$ (note that $\left.\varepsilon_{h^{-1} g h}=\varepsilon_{g}\right)$. Then

$$
\varphi(h) Y_{h^{-1} g h}(u, z) \varphi(h)^{-1}=Y_{g}(h u, z)
$$

on $V_{\Gamma_{l, g}+\beta_{g}}$ for $u \in V_{\Gamma_{l}}$. In particular, $\varphi(h)$ gives an isomorphism of $h^{-1} g h$-twisted modules $V_{\Gamma_{l, h^{-1} g h}+\beta_{h^{-1} g h}} \simeq h \circ V_{\Gamma_{l, g}+\beta_{g}}$.

Proof. Clearly, (6.12) holds for $u \in V_{L}$. We only need to prove the theorem for $u \in V_{L_{g}+\lambda_{l}}$. We first deal with the case $\varepsilon_{g}=0$. Take $u=l(a)=l\left(a_{g}\right) \in V_{L+\lambda_{l}}$. Since $\lambda_{l}(-1) \in V_{L}$ and $h \lambda_{l}(-1)=\lambda(-1)$, from $\varphi(h) Y_{h^{-1} g h}\left(\lambda_{l}(-1), z\right) \varphi(h)^{-1}=Y_{g}\left(\lambda_{l}(-1), z\right)$ we see that $\varphi(h) \lambda_{l}(m) \varphi(h)^{-1}=\lambda_{l}(m)$ for $m \in \mathbb{Z}$. Recall (3.17) and (3.18). Then as in the proof of Proposition 6.2 we have

$$
\begin{aligned}
\varphi(h) Y_{h^{-1} g h}\left(l\left(a_{h^{-1} g h}, z\right) \varphi(h)^{-1} b_{0, g}=\right. & E^{-}\left(-\lambda_{l}, z\right) z^{\left\langle\lambda_{l}, \bar{a}_{0, g}+\beta_{g}\right\rangle} \varphi(h) l\left(a_{h^{-1} g h} a_{0, h^{-1} g h}\right) \\
& \in V_{L_{g}+\lambda_{l}+\beta_{g}} .
\end{aligned}
$$

From Lemma 6.5, we have

$$
\varphi(h) Y_{h^{-1} g h}\left(l\left(c_{0, g}\right), z\right) \varphi(h)^{-1}=\lambda(g, h) Y_{g}\left(l\left(c_{0, g}\right), z\right) .
$$

Since $h\left(\bar{c}_{0, h^{-1} g h}(-1)\right)=\bar{c}_{0, g}(-1)$ in $V_{L}$, we see that

$$
\varphi(h) E^{ \pm}\left(-\bar{c}_{0, h^{-1} g h}, z\right) \varphi(h)^{-1}=E^{ \pm}\left(-\bar{c}_{0, g}, z\right)
$$

(cf. (6.7)) and that

$$
\varphi(h) c_{0, h^{-1} g h} \varphi(h)^{-1}=\lambda(g, h) \mathcal{c}_{0, g}
$$

on $V_{\Gamma_{l}+\beta_{g}}$. Thus

$$
\begin{aligned}
\varphi(h)\left(l\left(a_{g} c_{0, g}\right)\right) & =\varphi(h)(-1)^{\left\langle\lambda_{l}, \bar{c}_{0, g}\right\rangle} c_{0, g} b_{l, g} \\
& =(-1)^{\left\langle\lambda_{l}, \bar{c}_{0, g}\right\rangle} \lambda(g, h) c_{0, g} \varphi(h)\left(b_{l, g}\right)=l\left(a_{g} c_{0, g}\right),
\end{aligned}
$$

where we identify $l\left(a_{g}\right) \in V_{\Gamma_{l}}$ with $b_{l, g}$ introduced in (5.4). On the other hand,

$$
Y_{g}\left(h l\left(a_{g}\right), z\right) b_{0, g}=Y_{g}\left(l\left(a_{g}\right), z\right) b_{0, g}=E^{-}\left(-\lambda_{l}, z\right) z^{\left\langle\lambda_{l}, \bar{c}_{0, g}+\beta_{g}\right\rangle} l\left(a_{g} c_{0, g}\right) \in V_{L_{g}+\lambda_{l}+\beta_{g}} \text {. }
$$

That is,

$$
\varphi(h) Y_{h^{-1} g h}\left(l\left(a_{g}\right), z\right) \varphi(h)^{-1} b_{0, g}=Y_{g}\left(h_{l}\left(a_{g}\right), z\right) b_{0, g} .
$$


We already know from the proof of Theorem 5.7 that for any $v \in V_{L+\lambda_{l}}, Y_{g}(h v, z)$ (resp. $\left.Y_{h^{-1} g h}(v, z)\right)$ can be expressed in terms of $Y_{g}(h x, z)$ and $Y_{g}\left(h l\left(a_{g}\right), z\right)$ (resp. $Y_{h^{-1} g h}(x, z)$ and $\left.Y_{h^{-1} g h}\left(l\left(a_{g}\right), z\right)\right)$ for $x \in \mathfrak{g}$. Also $\varphi(h) Y_{h^{-1} g h}(x, z) \varphi(h)^{-1}=Y_{g}(h x, z)$. Thus (6.13) is true with $l\left(a_{g}\right)$ replaced by any $v \in V_{L+\lambda_{l}}$. Again as in the proof of Proposition 6.2 we use commutativity to show that (6.13) holds with $b_{0, g}$ replaced by any $v \in V_{L_{g}+\beta_{g}}$. Similarly for $v \in V_{L_{g}+\lambda_{l}+\beta_{g}}$.

The argument for $\varepsilon_{g}=1$ is similar to that for $\varepsilon_{g}=0$ if we notice that

$$
\varphi(h) c_{0, g} b=\lambda(h, g) c_{0, g} b \varphi(h)
$$

as operators on $V_{\Gamma_{l, g}+\beta_{g}}$, where $b \in \hat{L}_{g}$ such that $\bar{b}=2 h_{l, g}$ (cf. (5.5)).

Using the isomorphism $\sigma_{g}$ between $V_{\Gamma_{l}}$ and $V$ we define a linear map $Y: V \rightarrow($ End $V)\left[\left[z, z^{-1}\right]\right]$ by $Y(u, z)=\sigma_{1} Y(u, z) \sigma_{1}^{-1}$. For $g \in G$ we also have a linear map $Y_{g}: V \rightarrow($ End $V(g))\left[\left[z^{1 / N}, z^{-1 / N}\right]\right]$ by $Y_{g}(u, z)=\sigma_{g} Y\left(\sigma_{1}^{-1} u, z\right) \sigma_{g}^{-1}$. We immediately have:

Theorem 6.7. (1) The space $\left(V, Y, \mathbf{1}_{\mathbb{Z}+\frac{1}{2}}, \omega\right)$ is a holomorphic VOA isomorphic to $V_{\Gamma_{1}}$. Moreover, $G$ is a group of automorphisms of $V$.

(2) For $g \in G$ the space $V(g)$ is the unique irreducible $g$-twisted V-module.

(3) Let $g, h \in G$ and assume that $h\left(a_{l, h^{-1} g h}^{*}\right)=a_{l, g}^{*}$ if $\varepsilon_{g}=1$. Then there exists an invertible linear map $\varphi(h)$ from $V\left(h^{-1} g h\right)$ to $V(g)$ such that for $u \in V$,

$$
\varphi(h) Y_{h^{-1} g h}(u, z) \varphi(h)^{-1}=Y_{g}(h u, z) .
$$

In particular, $\varphi(h)$ gives an isomorphism of $h^{-1}$ gh-twisted $V$-modules $V\left(h^{-1} g h\right) \simeq$ $h \circ V(g)$.

\section{Modular Invariance}

In this section we assume that $l$ is divisible by 24 , and we let $V=V(1)$ be the holomorphic VOA constructed in the last section. We have previously considered finite subgroups $G<G L(l)$ acting on $V$ as described above (see in particular the paragraph following (4.2). In this section we always assume that $G<S O(l, \mathbb{R})<G L(l)$, in which case the action of $g \in G$ on $A$ is via the diagonal embedding $g \mapsto(g, g)$. Furthermore we assume the $G$ fixes a non-zero vector of $A^{+}$. We may now apply Theorem 6.7. So $G$ is a group of automorphisms of $V$ and for each $g \in G$ we have a representation of $C_{G}(g)$ on the unique irreducible $g$-twisted $V$-module $V(g)$. We will also be concerned with the VOA $C M^{0}\left(\mathbb{Z}+\frac{1}{2}\right)$ for which analogous statements hold thanks to Corollary 5.8.

The goal of this section is to establish the modular invariance properties $\mathrm{SH} 2$ and SH3 for $V$, and SR3 and SR4 for $V_{L}$. In fact a rather stronger formulation of SR 4 will be established. As we have mentioned in the introduction, the assumption that $24 \mid l$, rather than just $8 \mid l$ which we have assumed up till now, is made mainly so that the results of [M2] can be quoted directly. We leave it to the reader to reformulate the results of [M2] so that only the condition $8 \mid l$ is needed. The results of [M2] are used heavily throughout this section.

In order to deal with $V$ and its $g$-twisted modules $V(g)$, the main problem is to identify $V(g)$ with the graded space denoted by $\mathscr{H}_{g}$ in [M2]. So let us consider the 
underlying linear space of $V(g)$. Using (6.1) and (4.17) we have

$$
V(g) \simeq \Lambda^{0}\left(A^{-}\left(\mathbb{Z}+\frac{1}{2}, g\right)\right) \oplus N_{g} \otimes \Lambda^{\varepsilon_{g}}\left(A^{-}(\mathbb{Z}, g)\right),
$$

where $\varepsilon_{g}$ is as in (5.1) and where $N_{g}$ is the one-dimensional $C_{G}(g)$-module affording the character $\chi_{g}(6.2)$. Of course $V(g)$ is also graded as in Remark 4.2, and we shall return to this in due course.

Now from (4.12) we have for $g$ of order $N$,

$$
\Lambda^{\text {even }}\left(A^{-}\left(\mathbb{Z}+\frac{1}{2}, g\right)\right)=\Lambda^{\text {even }}\left(A_{N / 2, g}^{-}\right) \Lambda^{\text {even }}\left(S_{g}\right) \oplus \Lambda^{\text {odd }}\left(A_{N / 2, g}^{-}\right) \Lambda^{\text {odd }}\left(S_{g}\right),
$$

where $S_{g}$ is shorthand for $\sum_{n / N-1 / 2<0} A_{n, g} \otimes t^{(n / N-1 / 2)}$ and by convention $A_{N / 2}^{-}=0$ if $N$ is odd.

Now $C_{G}(g)$ acts on $A_{N / 2, g}$ via the diagonal embedding $C_{G}(g) \rightarrow S O\left(A_{N / 2, g}^{-}\right) \rightarrow$ $S O\left(A_{N / 2, g}\right)$ and hence (see Lemma 2.2 of [M2]) lifts to an embedding $C_{G}(g) \rightarrow \operatorname{Spin}\left(A_{N / 2, g}\right)$. Then it is well-known that the spaces $\Lambda^{\text {even }}\left(A_{N / 2, g}^{-}\right)$, $\Lambda^{\text {odd }}\left(A_{N / 2, g}^{-}\right)$are just the two half-spin modules for $\operatorname{Spin}\left(A_{N / 2, g}\right)$ which we denote by $\Delta_{1 / 2, g}^{+}, \Delta_{1 / 2, g}^{-}$to conform (more or less) to the notation of [M2]. Thus we may rewrite the first summand of (7.1), considered as a graded $C_{G}(g)$-module, as

$$
\Delta_{1 / 2, g}^{+} \Lambda^{\text {even }}\left(S_{g}\right) \oplus \Delta_{1 / 2, g}^{-} \Lambda^{\text {odd }}\left(S_{g}\right) \text {. }
$$

For $g \in G$ the function $B(g)$ is defined in [M2] as follows:

$$
B(g)=\frac{1}{2} \sum_{k=0}^{n-1} B_{2}(k / N) \operatorname{dim} A_{k, g}^{+},
$$

where $g$ has order $N$ and $B_{2}(x)$ is the second Bernoulli polynomial defined by $B_{2}(x)=x^{2}-x+1 / 6$.

Lemma 7.1. We have

$$
c_{Z, g}-l / 24=\left\{\begin{array}{lll}
B(g) & \text { if } & Z=\mathbb{Z} \\
\frac{1}{2} B\left(g^{2}\right)-B(g) & \text { if } & Z=\mathbb{Z}+1 / 2
\end{array} .\right.
$$

Proof. By (7.3) we get

$$
\begin{aligned}
B(g) & =1 / 2 \sum_{k=0}^{N-1}\left(k^{2} / N^{2}-k / N+1 / 6\right) \operatorname{dim} A_{k, g}^{+} \\
& =1 / 2 \sum_{k=0}^{N-1}\left((k / N-1 / 2)^{2}-1 / 12\right) \operatorname{dim} A_{k, g}^{+} \\
& =c_{\mathbb{Z}, g}-l / 24 .
\end{aligned}
$$

using (4.19) for $Z=\mathbb{Z}$. The case $Z=\mathbb{Z}+1 / 2$ is proved similarly.

Remark 7.2. By Proposition 4.12 (4), the Virasoro algebra is represented on each $C M^{r}(Z, g)$, hence on $V(g)$, with central charge $l$.

According to the general principle of (2.23), for modular invariance considerations we should grade $S_{g}$ in (7.2) positively by replacing $t^{-1}$ by $q$ and incorporate the $L(0)$-grading of Remark 4.2 (cf. (4.37) and (4.38)) as well as an overall grade shift by $q^{-l / 24}$. After doing this, we find using Lemma 7.1 that the (shifted) $q$-grading of 
the space (7.2) may be presented as

$$
q^{1 / 2 B\left(g^{2}\right)-B(g)}\left(\Delta_{1 / 2, g}^{+} \Lambda^{\text {even }}\left(S_{g}\right) \oplus \Delta_{1 / 2, g}^{-} \Lambda^{\text {odd }}\left(S_{g}\right)\right)
$$

as a $q$-graded $C_{G}(g)$-module. This is precisely the first summand of the space $\mathscr{H}_{g}$ of Theorem 10.2 of [M2].

We turn our attention to the second summand of (7.1). First suppose that $T$ is a $\mathbb{C} G$-module with a one-dimensional trivial $G$-submodule, say $T=1 \oplus U$. Then there are isomorphisms of (graded) $G$-modules

$$
\Delta^{\text {even }}(T) \simeq \Lambda^{\text {odd }}(T) \simeq \Lambda(U) .
$$

As $A^{-}(\mathbb{Z}, g)=A_{0, g}^{-} \oplus\left(\sum_{n<0} A_{n, g} \otimes t^{n / N)}\right.$ (see (4.13) and as $A_{0, g}^{-}$has a non-trivial $C_{G}(g)$-invariant by hypothesis then (7.5) applies to the action of $C_{G}(g)$ on $A^{-}(\mathbb{Z}, g)$. The upshot is that as graded $C_{G}(g)$-module the space $N_{g} \otimes \Lambda^{\varepsilon_{g}}\left(\Lambda^{-}(\mathbb{Z}, g)\right)$ is independent of the sign $\varepsilon_{g}$, and with an obvious formalism we may write this latter space as $1 / 2 N_{g} \otimes \Lambda\left(A^{-}(\mathbb{Z}, g)\right)$.

Arguing as before, we have

$$
\Lambda\left(A^{-}(\mathbb{Z}, g)\right) \simeq \Delta_{0, g} \otimes \Lambda\left(T_{g}\right),
$$

where $\Delta_{0, g}$ is the spin module for $\operatorname{Spin}\left(A_{0, g}\right)$ and $T_{g}=\sum_{n<0} A_{n, g} \otimes t^{n / N}$. Moreover the character $\chi_{g}$ of $C_{G}(g)$ used in (6.2) is seen to be precisely the character denoted by $\lambda(g, \cdot)$ in [M2] (see in particular Lemma 8.2 loc cit.). Finally, if we again incorporate the $q$-grading and the shift by $q^{-l / 24}$ we obtain, using Lemma 7.1, the space

$$
\frac{1}{2} q^{B(g)} N_{g} \otimes \Delta_{0, g} \otimes \Lambda\left(T_{g}\right)
$$

which is precisely the second summand of the space denoted $\mathscr{H}_{g}$ in [M2]. Consequently we have shown:

Proposition 7.3. Let $\mathscr{H}_{g}$ be the q-graded $C_{G}(g)$-module of Theorem 10.2 of [M2]. Then $\mathscr{H}_{g}$ is isomorphic, as graded $C_{G}(g)$-module, to the irreducible g-twisted $V$-module $V(g)$ graded by the conformal weight together with an overall graded shift of $q^{-l / 24}$.

Let us write $q^{-24 / l} V(g)$ for the $q$-graded $g$-twisted sector, and for $h \in C_{G}(g)$ set

$$
Z(g, h, \tau)=q^{-l / 24}(\text { graded trace of } h \text { on } V(g)) \text {. }
$$

Because of Proposition 7.3 we can apply Theorem 10.2 of [M2] to conclude

Theorem 7.4. For the holomorphic VOA $V$ and the group $G<S O(l, \mathbb{R})$ described above, properties $\mathrm{SH} 1-\mathrm{SH} 3$ hold. In particular we have the relation

$$
Z(g, h, \gamma \tau)=\sigma\left(\gamma^{-1}, g, h\right) Z((g, h) \gamma, \tau)
$$

for a certain 1-cocycle $\sigma$ of $\Gamma=S L(2, \mathbb{Z})$. Thus $\sigma$ satisfies for $\gamma_{1}, \gamma_{2} \in \Gamma, g h=h g$ :

$$
\sigma\left(\gamma_{1} \gamma_{2}, g, h\right)=\sigma\left(\gamma_{1},(g, h) \gamma_{2}^{-1}\right) \sigma\left(\gamma_{2}, g, h\right) \text {. }
$$

Now we turn our attention to the $C M^{0}\left(\mathbb{Z}+\frac{1}{2}\right)$. In this case there are, for $g \in G$, exactly four irreducible $g$-twisted modules $C M^{r}(Z, g)$ for $r=0,1 Z=\mathbb{Z}$ or $\mathbb{Z}+\frac{1}{2}$ and SR1, SR2 hold (cf. Corollary 5.8). We are going to show that SR3, SR4 of Sect. 2 also hold. To be more precise, let $H$ be the vector space with basis indexed by the functions $Z(g, h, \tau)$ of the VOA $V$. Then $H$ affords a representation of $S L(2, \mathbb{Z})$ via

$$
\gamma: Z(g, h, \tau) \mapsto Z(g, h, \gamma \tau)
$$


by (7.8). Next note that if (2.25) is to hold in general, then in particular we have

$$
Z^{i}(1,1, \gamma \tau)=\sum_{j} a_{i j} Z^{j}(1,1, \tau),
$$

where in (7.9) $Z^{i}(1,1, \tau)$ is just the character of the $\mathrm{i}^{\text {th }}$ irreducible $C M\left(\mathbb{Z}+\frac{1}{2}\right)$ module. In the case of affine Kac-Moody algebras this action of the modular group on the space of the irreducible characters is understood [KP]. In particular, if we order the irreducible $C M\left(\mathbb{Z}+\frac{1}{2}\right)$-modules as follows: $C M^{0}\left(\mathbb{Z}+\frac{1}{2}\right), C M^{1}\left(\mathbb{Z}+\frac{1}{2}\right)$, $C M^{0}(\mathbb{Z}), C M^{1}(\mathbb{Z})$. Then the matrix representations $S=\left(\begin{array}{rr}0 & -1 \\ 1 & 0\end{array}\right)$ and $T=\left(\begin{array}{ll}1 & 1 \\ 0 & 1\end{array}\right)$ are as follows:

$$
S=\left(\begin{array}{rrrr}
\frac{1}{2} & \frac{1}{2} & \frac{1}{2} & \frac{1}{2} \\
\frac{1}{2} & \frac{1}{2} & -\frac{1}{2} & -\frac{1}{2} \\
\frac{1}{2} & -\frac{1}{2} & \frac{1}{2} & -\frac{1}{2} \\
\frac{1}{2} & -\frac{1}{2} & -\frac{1}{2} & \frac{1}{2}
\end{array}\right), \quad T=\left(\begin{array}{rrrr}
1 & 0 & 0 & 0 \\
0 & -1 & 0 & 0 \\
0 & 0 & 1 & 0 \\
0 & 0 & 0 & 1
\end{array}\right) .
$$

We shall prove this below, but it is well-known. Let $H_{0}$ be the four-dimensional space affording this representation of $S L(2, \mathbb{Z})$. We shall prove

Theorem 7.5. The representation of $S L(2, \mathbb{Z})$ on the space of functions $Z^{i}(g, h, \tau)$ associated with $G$ and $C M^{0}\left(\mathbb{Z}+\frac{1}{2}\right)$ is the tensor product $H \otimes H_{0}$ of the "holomorphic" space $H$ of $V$ and the space of characters $H_{0}$.

This means that if $\gamma \in S L(2, \mathbb{Z})$ and $\gamma$ is represented on $H_{0}$ as the $4 \times 4$ matrix $\left(m_{i j}\right)$, then

$$
Z^{i}(g, h, \gamma \tau)=\sum_{j=1}^{4} m_{i j} Z^{j}\left(g^{a} h^{c}, g^{b} h^{d}, \tau\right)
$$

Since $\sigma$ is a 1-cocycle it is enough to establish (7.11) for $S$ and $T$ as they generate $S L(2, \mathbb{Z})$. Establishing (7.11) is easy for $\gamma=T$, and we leave this to the reader in order to concentrate on the case $\gamma=S$.

We introduce the linear transformation $z$ of $A$ which acts as - 1. Of course $z$ is not an element of our group $G$, nevertheless $z$ induces an automorphism of $C M\left(\mathbb{Z}+\frac{1}{2}\right)$ which we still denote by $z$. From (4.18) the following is clear:

$$
c_{\mathbb{Z}, g}=c_{\mathbb{Z}+\frac{1}{2}, g z} .
$$

Unlike the proof of Theorem 7.3, the proof of Theorem 7.5 does not follow immediately from [M2], so we must adopt an approach which is less direct. We will establish that the last two columns of the $S$-matrix in (7.10) are correct, that is we have

$$
Z^{i}(g, h, S \tau)=\frac{1}{2} \sigma\left(S^{-1}, g, h\right)\left(Z^{1}\left(h, g^{-1}, \tau\right)-Z^{2}\left(h, g^{-1}, \tau\right)\right),
$$

where $i=3$ or 4 , so that $Z^{i}(g, h, \tau)$ corresponds to the graded trace of $h$ on $C M^{r}(\mathbb{Z}, g)$ (recall that the isomorphism of $C M^{0}(\mathbb{Z}, g)$ and $C M^{1}(\mathbb{Z}, g)$ as graded $C_{G}(g)$-modules was established prior to Proposition 7.3).

First from (7.6), $Z^{i}(g, h, \tau) \equiv 0$ precisely when $h$ has an eigenvector in $A_{0, g}$ with eigenvalue -1 , that is when $\langle g, h z\rangle$ fixes a non-zero vector of $A$. But then of course $g^{-1}$ fixes a non-zero vector of the (-1)-eigenspace of $h$ of $A$ and so again 
$C M^{0}\left(\mathbb{Z}+\frac{1}{2}, h\right)$ and $C M^{1}\left(\mathbb{Z}+\frac{1}{2}, h\right)$ are isomorphic $\langle g\rangle$-modules. So in this case both sides of (7.13) are equal to 0 . So we may assume that $h z$ fixes no non-zero vector on $A_{0, g}$.

Now use (7.7) to get

$$
2 Z^{i}(g, h, \tau)=q^{B(g)} \operatorname{tr}_{N_{g}}(h) \operatorname{tr}_{\Delta_{0, g}}(h)\left(\text { trace } h \text { on } \Lambda\left(T_{g}\right)\right)
$$

with $\Lambda\left(T_{g}\right)=\Lambda\left(\sum_{n>0} A_{n, g} q^{n / N}\right)(N=$ order of $g)$. To compare with [M2], let $\left(x_{i}, y_{i}\right)$ be such that $\left(e^{2 \pi i x_{i}}, e^{2 \pi i y_{l}}\right)$ are the simultaneous eigenvalues of $(g, h)$ on $A$ with $0<x_{i} \leqq 1$. Then the trace of $h$ on $\Lambda\left(T_{g}\right)$ is equal to

$$
\begin{aligned}
\prod_{n \geqq 0, n \in \mathbb{Z}} & \prod_{0<x_{j} \leqq 1 / 2}\left(1+q^{n+x_{J}} e^{2 \pi i y_{J}}\right)\left(1+q^{n+1-x_{J}} e^{-2 \pi i y_{J}}\right) . \\
& \cdot \prod_{n>0, n \in \mathbb{Z}} \prod_{x_{j}=0}\left(1+q^{n} e^{2 \pi i y_{j}}\right)\left(1+q^{n} e^{-2 \pi i y_{j}}\right) \\
= & \eta(\tau)^{2 l} q^{-1 / 2 \sum_{j} B_{2}\left(x_{j}\right)} . \\
& \cdot \prod_{0<x_{j} \leqq 1 / 2} \eta(\tau)^{-2} q^{1 / 2 B_{2}\left(x_{j}\right)} \prod_{n \geqq 0}\left(1+q^{n+x_{j}} e^{2 \pi i y_{j}}\right)\left(1+q^{n+1-x_{j}} e^{-2 \pi i y_{j}}\right) . \\
& \cdot \prod_{x_{j}=0} \eta(\tau)^{-2} q^{1 / 12} \prod_{n>0}\left(1+q^{n} e^{2 \pi i y_{j}}\right)\left(1+q^{n} e^{-2 \pi i y_{j}}\right) .
\end{aligned}
$$

If we replace $h$ by $h z$ then all the signs in the brackets change from + to - . Then if we compare with Eqs. (5.6), (5.7), (6.1) of [M2], we see that the whole product is equal to

$$
q^{-1 / 2 \sum_{j} B_{2}\left(x_{J}\right)} f_{A}(g, h z, \tau)
$$

in the notation of Chapter 6 [loc cit]. Using (7.3) we have established the equality

$$
2 Z^{i}(g, h, \tau)=\operatorname{tr}_{N_{g}}(h) \operatorname{tr}_{\Delta_{0, g}}(h) f_{A}(g, h z, \tau) .
$$

we are going to use Theorem 6.2 of [M2]. It tells us that $f_{A}(g, h z, S \tau)=f_{A}\left(\left(h z, g^{-1}\right) S^{-1}, S \tau\right)=c\left(S,\left(h z \cdot g^{-1}\right)\right)^{-1} t\left(S,\left(h z, g^{-1}\right)\right)^{-1} f_{A}(h z, g, \tau)$ i.e.,

$$
f_{A}(g, h z, S \tau)=c\left(S^{-1},(g, h z)\right) t\left(S^{-1},(g, h z)\right) f_{A}(h z, g, \tau)
$$

(since $c$ and $t$ are 1-cocycles - see Lemma 5.3 and Theorem 5.7 of [M2]). Moreover, we similarly get

$$
\begin{gathered}
f_{A}\left(h z, g^{-1}, \tau\right)=q^{B(h z)} \prod_{0<x_{j}^{\prime} \leqq 1 / 2} \prod_{n \geqq 0}\left(1-q^{n+x_{j}^{\prime}} e^{2 \pi i y_{j}^{\prime}}\right)\left(1-q^{n+1-x_{j}^{\prime}} e^{-2 \pi i y_{j}^{\prime}}\right) . \\
\cdot \prod_{x_{j}^{\prime}=0} \prod_{n>0}\left(1-q^{n} e^{2 \pi i y_{j}^{\prime}}\right)\left(1-q^{n} e^{-2 \pi i y_{j}^{\prime}}\right)
\end{gathered}
$$

where $\left(e^{2 \pi i x_{j}^{\prime}}, e^{2 \pi i y_{j}^{\prime}}\right)$ are simultaneous eigenvalues for $\left(h z, g^{-1}\right)$ on $A$ with $0<x_{j}^{\prime} \leqq 1$.

Next note that from Lemma 7.1 and (7.12) we get

$$
B(h z)=B\left(h^{2}\right) / 2-B(h) .
$$

Then (7.16) is equal to

$$
q^{B\left(h^{2}\right) / 2-B(h)}\left(\operatorname{tr} g^{-1} z \text { on } \Lambda_{S_{h}}\right)=\operatorname{tr}_{\Delta_{1 / 2, h}}\left(g^{-1} z\right)^{-1}\left(\operatorname{tr} g^{-1} z \text { on } C M\left(\mathbb{Z}+\frac{1}{2}, h\right)\right) \text {, }
$$


as we see by the argument leading to (7.2). But $z$ acts as 1 on $\Delta_{1 / 2, h}^{+}$and -1 on $\Delta_{1 / 2, h}^{-}$(unless this latter space is 0 ), so (7.17) becomes

$$
\operatorname{tr}_{\Delta_{1 / 2, h}}\left(g^{-1} z\right)^{-1}\left(Z^{1}\left(h, g^{-1}, \tau\right)-Z^{2}\left(h g^{-1}, \tau\right)\right) \text {. }
$$

Putting everything together, we obtain:

$$
\begin{gathered}
Z^{i}(g, h, S \tau)=1 / 2 \operatorname{tr}_{N_{g}}(h) \operatorname{tr}_{\Delta_{0, g}}(h) c\left(S^{-1}, g, h z\right) t\left(S^{-1}, g, h z\right) \\
\operatorname{tr}_{\Delta_{1 / 2, h}}\left(g^{-1} z\right)^{-1}\left(Z^{1}\left(h, g^{-1}, \tau\right)-Z^{2}\left(h, g^{-1}, \tau\right)\right)
\end{gathered}
$$

that is, (7.13) is correct up to a constant which we still need to identify. One can calculate it directly from (7.18) and [M2], but it is enough to observe that it must be as desired because by Theorem 7.4 we have

$$
Z^{1}(g, h, S \tau)+Z^{i}(g, h, S \tau)=\sigma\left(S^{-1}, g, h\right)\left(Z^{1}\left(h, g^{-1}, \tau\right)+Z^{i}\left(h, g^{-1}, \tau\right)\right) .
$$

Now (7.13) follows, and Theorem 7.5 follows from this and (7.18).

Finally, we consider the so-called 1-loop characters. For $g \in G$ let $\alpha_{g}$ range over the irreducible characters of $C_{G}(g)$. For the holomorphic VOA $V$ we can decompose the (grade-shifted) irreducible $g$-twisted module $q^{-l / 24} V(g)$ into irreducible $C_{G}(g)$-submodules. So formally we get

$$
q^{-l / 24} V(g)=\oplus_{\alpha_{g}}\left(\alpha_{g} \otimes \phi_{\alpha_{g}}(q)\right),
$$

where $\phi_{\alpha_{g}}(q)$ is the "graded multiplicities," or 1-loop character, corresponding to $\alpha_{g}$. One expects that $\phi_{\alpha_{g}}(q)$ is the character of an irreducible $V^{G}$-module, but this is unknown. Elementary character theory yields

$$
\phi_{\alpha_{g}}(q)=\frac{1}{\left|C_{G}(g)\right|} \sum_{h \in C_{G}(g)} Z(g, h, \tau) \overline{\alpha_{g}(h)},
$$

so the 1-loop characters form a basis of $H$ if we choose (as we do) representatives $g$ of each conjugacy class of $G$.

Similarly in the case of the VOA $C M^{0}\left(\mathbb{Z}+\frac{1}{2}\right)$ we get 1 -loop characters

$$
\phi_{\alpha_{g}}^{i}(q)=\frac{1}{\left|C_{G}(g)\right|} \sum_{h \in C_{G}(g)} Z^{i}(g, h, \tau) \overline{\alpha_{g}(h)}
$$

for $i=1,2,3,4$ in an obvious sense.

Define a hermitian form $\langle\cdot, \cdot\rangle$ on either $H$ or $H \otimes H_{0}$ by taking the $\phi_{\alpha_{g}}$ or $\phi_{\alpha_{g}}^{i}$ to be an orthonormal basis.

Theorem 7.6. The representation of $S L(2, \mathbb{Z})$ on either $H$ or $H \otimes H_{0}$ is unitary with respect to $\langle\cdot, \cdot\rangle$, and moreover, $T$ is diagonal and $S$ symmetric if they are represented as matrices with respect to the basis of 1-loop characters.

Proof. By Theorem 9.1 of [M2] we find that the 1-cocycle $\sigma\left(\gamma^{-1}, g, h\right)$ is symmetric if $\gamma=S$. Moreover $\sigma\left(T^{-1}, g, h\right)$ is independent of $h$.

Using these facts, the theorem can be established on a purely group-theoretic basis by several applications of the orthogonality relations. We omit details as the result is also discussed, in greater generality, in Sect. 4.b. of [DVVV]. 


\section{References}

[Bal] Bantay, P.: Orbifolds and Hopf algebras. Phys. Letter B 245, No. 3, 4 (1990)

[Ba2] Bantay, P.: Orbifolds, Hopf algebras, and the Moonshine module. Letter Math. Phys. 22, 187-194 (1991)

[Bo] Borcherds, R.E.: Vertex algebras, Kac-Moody algebras and the Monster. Proc. Natl. Acad. Sci. USA 83, 3068-3071 (1986)

[DVVV] Dijkgraaf, R., Vafa, C., Verlinde, E., Verlinde, H.: The operator algebra of orbifold models. Comm. Math. Phys. 123, 485-526 (1989)

[DHVW] Dixon, L., Harvey, J., Vafa, C., Witten, E.: Strings on orbifolds I, II. Nucl. Phys. B 273, 678 (1985); Nucl. Phys. B 274, 285 (1986)

[DGH] Dixon, L., Ginsparg, P., Harvey, J.: Beauty and the beast: Superconformal symmetry in a Monster module. Commun. Math. Phys. 119, 285 (1988)

[D1] Dong, C.: Vertex algebras associated with even lattices. J. Algebra 161, 245-265 (1993)

[D2] Dong, C.: Twisted modules for vertex algebras associated with even lattices. J. Algebra 164 (1994)

[DL1] Dong, C., Lepowsky, J.: Generalized Vertex Algebras and Relative Vertex Operators. Progress in Math. Boston: Birkhäuser, vol. 112, 1993

[DL2] Dong, C., Lepowsky, J.: The algebraic structure of relative twisted vertex operators. To appear

[F] Frenkel, I.B.: Two constructions of affine Lie algebra represntations and bosonfermion correspondence in quantum field theory. J. Funct. Anal. 44, 259-327 (1981)

[FF] Feingold, A.J., Frenkel, I.B.: Classical affine Lie algebras. Adv. Math. 56, 117-172 (1985)

[FFR] Feingold, A.J., Frenkel, I.B., Ries, J.F.X.: Spinor construction of vertex operator algebras, triality and $E_{8}^{(1)}$. Contemp. Math. 121, (1991)

[FHL] Frenkel, I.B., Huang, Y.-Z., Lepowsky, J.: On axiomatic approaches to vertex operator algebras and modules. Preprint, 1989; Mem. Am. Math. Soc. 104, (1993)

[FLM1] Frenkel, I.B., Lepowsky, J., Meurman, A.: Vertex operator calculus. In: Mathematical Aspects of String Theory, Proc. 1986 Conference, San Diego, ed. by Yau, S.-T., Singapore: World Scientific, 1987, pp. 150-188

[FLM2] Frenkel, I.B., Lepowsky, J., Meurman, A.: Vertex Operator Algebras and the Monster. Pure and Applied Math. 134, London-New York: Academic Press, 1988

[K] Kac, V.G.: Infinite-dimensional Lie algebras, 3rd ed., Cambridge: Cambridge Univ. Press, 1990

[KP] Kac, V.G., Peterson, D.H.: Infinite-dimensional Lie algebras, theta functions and modular forms. Adv. Math. 53, 125-264 (1984)

[L] Lepowsky, J.: Calculus of twisted vertex operators. Proc. Natl. Acad Sci. USA 82, 8295-8299 (1985)

[LW] Lepowsky, J., Wilson, R.L.: The structure of standard modules, I: Universal algebras and the Rogers-Ramanujan identities. Invent. Math. 79, 199-290 (1984)

[M1] Mason, G.: $G$-elliptic systems and the genus zero problem for $M_{24}$. Bull. Am. Math. Soc. 25, No. 1, 45-53 (1991)

[M2] Mason, G.: Hecke operators and conformally-invariant orbifold models. To appear

[M3] Mason, G.: Finite group and modular functions. Proc. Symp. in Pure Math. 47, $181-210(1987)$

[S] Serre, J.P.: Cours d'arithmétique. Presses Universitaries de France, 1970

[T] Tsukada, H.: Shifted vertex operator algebras and $G$-elliptic systems. J. Math. Phys. 33, 2546-2556 (1992)

[Tu] Tuite, M.: Monstrous Moonshine from orbifolds. Preprint, DIAS-STP-90-14 
